\title{
Scaling limit of isoradial dimer models and the case of triangular quadri-tilings
}

\author{
Béatrice de Tilière ${ }^{1}$ \\ Institut für Mathematik, Universität Zürich, Winterthurerstrasse 190, 8057 Zürich, Switzerland
}

Received 13 March 2006; accepted 19 October 2006

Available online 29 January 2007

\begin{abstract}
We consider dimer models on planar graphs which are bipartite, periodic and satisfy a geometric condition called isoradiality, defined in [R. Kenyon, The Laplacian and Dirac operators on critical planar graphs, Invent. Math. 150 (2) (2002) 409-439]. We show that the scaling limit of the height function of any such dimer model is a Gaussian free field. Triangular quadri-tilings were introduced in [B. de Tilière, Quadri-tilings of the plane, math.PR/0403324, Probab. Theory Related Fields, in press]; they are dimer models on a family of isoradial graphs arising from rhombus tilings. By means of two height functions, they can be interpreted as random interfaces in dimension $2+2$. We show that the scaling limit of each of the two height functions is a Gaussian free field, and that the two Gaussian free fields are independent.

() 2006 Elsevier Masson SAS. All rights reserved.

\section{Résumé}

On considère le modèle de dimères sur des graphes planaires, bipartis, périodiques et satisfaisant une condition géométrique appelée isoradialité, définie dans [R. Kenyon, The Laplacian and Dirac operators on critical planar graphs, Invent. Math. 150 (2) (2002) 409-439]. Nous montrons que la limite d'échelle de la fonction de hauteur d'un tel modèle est décrite par un champ libre gaussien. Les quadri-pavages triangulaires ont été introduits dans [B. de Tilière, Quadri-tilings of the plane, math.PR/0403324, Probab. Theory Related Fields, in press]; ils constituent une famille de modèles de dimères sur des graphes engendrés par des pavages par losanges. Grâce à deux fonctions de hauteur, ils sont interprétés comme des interfaces discrètes en dimension $2+2$. Nous montrons que la limite d'échelle de chacune des fonctions de hauteur est décrite par un champ libre gaussien, et que ces deux champs libres sont indépendants.
\end{abstract}

() 2006 Elsevier Masson SAS. All rights reserved.

Keywords: Dimer model; Gausian free field; Scaling limit; Height function; Quadri-tilings

E-mail address: beatrice.detiliere@math.unizh.ch (B. de Tilière).

1 Supported by Swiss National Fund grant 47102009. 


\section{Introduction}

\subsection{Height fluctuations for isoradial dimer models}

\subsubsection{Dimer models}

The setting for this paper is the dimer model. It is a statistical mechanics model representing diatomic molecules adsorbed on the surface of a crystal. An interesting feature of the dimer model is that it is one of the very few statistical mechanics models where exact and explicit results can be obtained, see [9,10] for an overview. Another very interesting aspect is the alleged conformal invariance of its scaling limit, which is already proved in the domino and $60^{\circ}$-rhombus cases $[11,12,14]$. Theorem 1 of this paper shows this property for a wide class of dimer models containing the above two cases.

In order to give some insight, let us precisely define the setting. The dimer model is in bijection with a mathematical model called the 2-tiling model representing discrete random interfaces. The system considered for a 2-tiling model is a planar graph $G$. Configurations of the system, or 2-tilings, are coverings of $G$ with polygons consisting of pairs of edge-adjacent faces of $G$, also called 2-tiles, which leave no hole and don't overlap. The system of the corresponding dimer model is the dual graph $G^{*}$ of $G$. Configurations of the dimer model are perfect matchings of $G^{*}$, that is set of edges covering every vertex exactly once. Perfect matchings of $G^{*}$ determine 2-tilings of $G$ as explained by the following correspondence. Denote by $f^{*}$ the dual vertex of a face $f$ of $G$, and consider an edge $f^{*} g^{*}$ of $G^{*}$. We say that the 2-tile of $G$ made of the adjacent faces $f$ and $g$ is the 2-tile corresponding to the edge $f^{*} g^{*}$. Then 2-tiles corresponding to edges of a dimer configuration form a 2-tiling of $G$. Let us denote by $\mathcal{M}\left(G^{*}\right)$ the set of dimer configurations of $G^{*}$.

As for all statistical mechanics models, dimer configurations are chosen with respect to the Boltzmann measure defined as follows. Suppose that the graph $G^{*}$ is finite, and that a positive weight function $v$ is assigned to edges of $G^{*}$, then each dimer configuration $M$ has an energy, $\mathcal{E}(M)=-\sum_{e \in M} \log v(e)$. The probability of occurrence of the dimer configuration $M$ chosen with respect to the Boltzmann measure $\mu^{1}$ is:

$$
\mu^{1}(M)=\frac{\mathrm{e}^{-\mathcal{E}(M)}}{Z\left(G^{*}, v\right)}=\frac{\prod_{e \in M} \nu(e)}{Z\left(G^{*}, v\right)},
$$

where $Z\left(G^{*}, v\right)$ is the normalizing constant called the partition function. Using the bijection between dimer configurations and 2-tilings, $v$ can be seen as weighting 2-tiles, and $\mu^{1}$ as a measure on 2-tilings of $G$. When the graph $G^{*}$ is infinite, a Gibbs measure is defined to be a probability measure on $\mathcal{M}\left(G^{*}\right)$ with the following property: if the matching in an annular region is fixed, then matchings inside and outside of the annulus are independent, moreover the probability of any interior matching $M$ is proportional to $\prod_{e \in M} v(e)$. From now on, let us assume that the graph $G$ satisfies condition (*) below:

$(*)$

The graph $G$ is infinite, planar, and simple ( $G$ has no loops and no multiple edges); its vertices are of degree $\geqslant 3 . G$ is simply connected, i.e. it is the one-skeleton of a simply connected union of faces; and it is made of finitely many different faces, up to isometry.

\subsubsection{Isoradial dimer models}

This paper actually proves conformal invariance of the scaling limit for a sub-family of all dimer models called isoradial dimer models, introduced by Kenyon in [13]. Much attention has lately been given to isoradial dimer models because of a surprising feature: many statistical mechanics quantities can be computed in terms of the local geometry of the graph. This fact was conjectured in [13], and proved in [4]. The motivation for their study is further enhanced by the fact that the yet classical domino and $60^{\circ}$-rhombus tiling models are examples of isoradial dimer models. Last but not least their understanding allows us to apprehend a random interface model in dimension $2+2$ called the triangular quadri-tiling model introduced in [3], see Section 1.2.1.

Let us now define isoradial dimer models. Speaking in the terminology of 2-tilings, isoradial 2-tiling models are defined on graphs $G$ satisfying a geometric condition called isoradiality: all faces of an isoradial graph are inscribable in a circle, and all circumcircles have the same radius, moreover all circumcenters of the faces are contained in the closure of the faces. The energy of configurations is determined by a specific weight function called the critical weight function, see Section 2.1 for definition. Note that if $G$ is an isoradial graph, an isoradial embedding of the 
dual graph $G^{*}$ is obtained by sending dual vertices to the center of the corresponding faces. Hence, the corresponding dimer model is called an isoradial dimer model.

\subsubsection{Height functions}

Let $G$ be an isoradial graph, whose dual graph $G^{*}$ is bipartite. Then, by means of the height function, 2-tilings of $G$ can be interpreted as random discrete 2-dimensional surfaces in a 3-dimensional space that are projected orthogonally to the plane. In physics terminology, one speaks of random interfaces in dimension $2+1$. The height function, denoted by $h$, is an $\mathbb{R}$-valued function on the vertices of every 2-tiling of $G$, and is defined in Section 3.

\subsubsection{Gaussian free field in the plane}

The Gaussian free field in the plane is defined in Section 4. It is a random distribution which assigns to functions $\varphi_{1}, \ldots, \varphi_{k} \in C_{c, 0}^{\infty}\left(\mathbb{R}^{2}\right)$ (the set of compactly supported smooth functions of $\mathbb{R}^{2}$, which have mean 0 ), a real Gaussian random vector $\left(F \varphi_{1}, \ldots, F \varphi_{k}\right)$ whose covariance function is given by

$$
\mathbb{E}\left(F \varphi_{i} F \varphi_{j}\right)=\iint_{\mathbb{R}^{2}} g(x, y) \varphi_{i}(x) \varphi_{j}(y) \mathrm{d} x \mathrm{~d} y,
$$

where $g(x, y)=-\frac{1}{2 \pi} \log |x-y|$ is the Green function of the plane (defined up to an additive constant).

\subsubsection{Statement of result}

Let $G$ be an isoradial graph, whose dual graph $G^{*}$ is bipartite. Suppose moreover that $G^{*}$ is doubly periodic, i.e. that the graph $G^{*}$ and its vertex-coloring are periodic. Then by Sheffield's theorem [17], there exists a twoparameter family of translation invariant, ergodic Gibbs measures. Note that the two parameters of the Gibbs measures correspond to the normalized height change (horizontal and vertical) of dimer configurations on a family of toroidal graphs, which form a natural exhaustion of $G^{*}$. Let us denote by $\mu$ the unique measure of this two parameter family which has minimal free energy per fundamental domain. From now on, we assume that dimer configurations of $G^{*}$ are chosen with respect to the measure $\mu$.

Let us multiply the edge-lengths of the graph $G$ by $\varepsilon>0$, this yields a new graph $G^{\varepsilon}$. Let $h^{\varepsilon}$ be the unnormalized height function on 2-tilings of $G^{\varepsilon}$. An important issue in the study of the dimer model is the understanding of the fluctuations of $h^{\varepsilon}$, as the mesh $\varepsilon$ tends to 0 . This question is answered by Theorem 1 below. Define:

$$
\begin{aligned}
H^{\varepsilon}: C_{c, 0}^{\infty}\left(\mathbb{R}^{2}\right) & \longmapsto \mathbb{R}, \\
\varphi & \longmapsto H^{\varepsilon} \varphi=\varepsilon^{2} \sum_{v \in V\left(G^{\varepsilon}\right)} a\left(v^{*}\right) \varphi(v) h^{\varepsilon}(v),
\end{aligned}
$$

where $V\left(G^{\varepsilon}\right)$ denotes the set of vertices of the graph $G^{\varepsilon}$, and $a\left(v^{*}\right)$ is the area in $G^{*}$ of the dual face $v^{*}$ of a vertex $v$.

Theorem 1. Consider a graph $G$ satisfying the above assumptions, then $H^{\varepsilon}$ converges weakly in distribution to $\frac{1}{\sqrt{\pi}}$ times a Gaussian free field, that is for every $\varphi_{1}, \ldots, \varphi_{k} \in C_{c, 0}^{\infty}\left(\mathbb{R}^{2}\right),\left(H^{\varepsilon} \varphi_{1}, \ldots, H^{\varepsilon} \varphi_{k}\right)$ converges in law (as $\left.\varepsilon \rightarrow 0\right)$ to $\frac{1}{\sqrt{\pi}}\left(F \varphi_{1}, \ldots, F \varphi_{k}\right)$, where $F$ is a Gaussian free field.

- As a direct consequence of Theorem 1, we obtain convergence of the height function of domino and $60^{\circ}$-rhombus tilings chosen with respect to the uniform measure to a Gaussian free field. Note that this result is slightly different than those of $[11,12,14]$ since we work on the whole plane, and not on simply connected regions.

- The method for proving Theorem 1 is essentially that of [11], except Lemma 19 which is new. Nevertheless, since we work with a general isoradial graph (and not the square lattice), each step is adapted in a non-trivial way.

\subsection{The case of triangular quadri-tilings}

\subsubsection{Triangular quadri-tiling model}

An exciting consequence of Theorem 1 is that it allows us to understand height fluctuations in the case of a random interface model in dimension $2+2$, called the triangular quadri-tiling model. It is the first time this type of result can be obtained on such a model. 
$\mathrm{T}$ is a 2-tiling of $L(\mathrm{~T})$
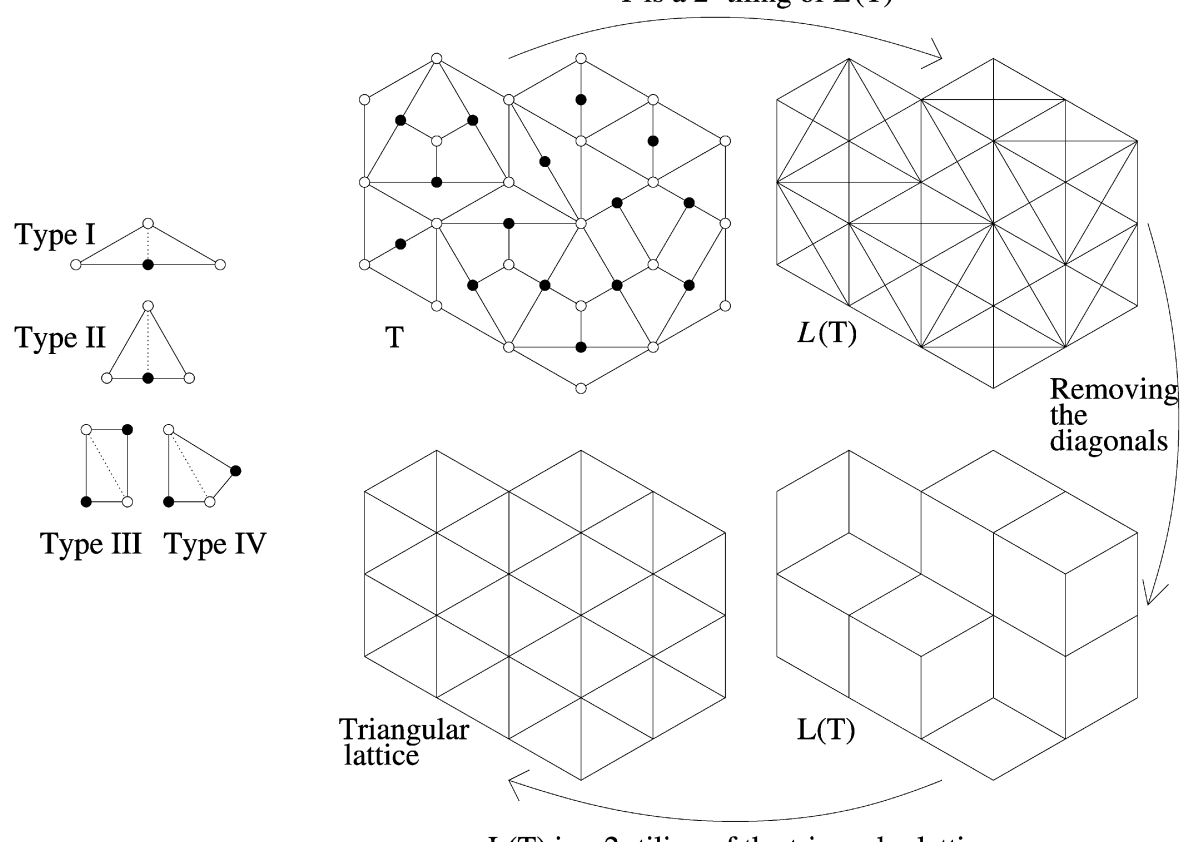

the

Type III Type IV

$\mathrm{L}(\mathrm{T})$ is a 2-tiling of the triangular lattice

Fig. 1. Four type of quadri-tiles (left). Triangular quadri-tilings correspond to two superimposed dimer models (right).

Let us start by defining triangular quadri-tilings. Consider the set of right triangles whose hypotenuses have length 1 , and whose interior angle is $\pi / 3$. Color the vertex at the right angle black, and the other two vertices white. A quadri-tile is a quadrilateral obtained from two such triangles in two different ways: either glue them along a leg of the same length matching the black (white) vertex to the black (white) one, or glue them along the hypotenuse. There are four types of quadri-tiles classified as I, II, III, IV, each of which has four vertices, see Fig. 1 (left). A triangular quadri-tiling of the plane is an edge-to-edge tiling of the plane by quadri-tiles that respects the coloring of the vertices, see $T$ of Fig. 1 for an example. Let $\mathcal{Q}$ denote the set of all triangular quadri-tilings of the plane, up to isometry.

In [3], triangular quadri-tilings of $\mathcal{Q}$ are shown to correspond to two superimposed dimer models in the following way, see also Fig. 1. Define a lozenge to be a $60^{\circ}$-rhombus. Then triangular quadri-tilings are 2 -tilings of a family of graphs $\mathcal{L}$ which are lozenge-with-diagonals tilings of the plane, up to isometry. Indeed let $T \in \mathcal{Q}$ be a triangular quadri-tiling, then on every quadri-tile of $T$ draw the edge separating the two right triangles, this yields a lozengewith-diagonals tiling $L(T)$ called the underlying tiling. Moreover, the lozenge tiling $L(T)$ obtained from $L(T)$ by removing the diagonals, is a 2-tiling of the equilateral triangular lattice $\mathbb{T}$.

Note that lozenge-with-diagonals tilings and the equilateral triangular lattice are isoradial graphs. Assigning the critical weight function to edges of their dual graphs, we deduce that triangular quadri-tilings of $\mathcal{Q}$ correspond to two superimposed isoradial dimer models.

\subsubsection{Height functions for triangular quadri-tilings}

Triangular quadri-tilings are characterized by two height functions in the following way. Let $T \in \mathcal{Q}$ be a triangular quadri-tiling, then the first height function, denoted by $h_{1}$, assigns to vertices of $T$ the "height" of $T$ interpreted as a 2-tiling of its underlying lozenge-with-diagonals tiling $L(T)$. The second height function, denoted by $h_{2}$, assigns to vertices of $T$ the height of $\mathrm{L}(T)$ interpreted as a 2-tiling of $\mathbb{T}$. An example of computation is given in Section 3.3. By means of $h_{1}$ and $h_{2}$, triangular quadri-tilings are interpreted in [3] as discrete random 2-dimensional surfaces in a 4-dimensional space that are projected orthogonally to the plane, i.e. in physics terminology, as random interfaces in dimension $2+2$. 


\subsubsection{Statement of result}

The notion of Gibbs measure can be extended naturally to the set $\mathcal{Q}$ of all triangular quadri-tilings, see Section 2.4. In [4], we give an explicit expression for such a Gibbs measure $\mathbb{P}$, and conjecture it to be of minimal free energy per fundamental domain among a four-parameter family of translation invariant, ergodic Gibbs measures. Let us assume that triangular quadri-tilings of $\mathcal{Q}$ are chosen with respect to the measure $\mathbb{P}$.

Corollary 2 below describes the fluctuations of the unnormalized height functions $h_{1}^{\varepsilon}$ and $h_{2}^{\varepsilon}$. Suppose that the equilateral triangular lattice has edge-lengths 1 , and let $\mathbb{T}^{\varepsilon}$ be the lattice $\mathbb{T}$ whose edge-lengths have been multiplied by $\varepsilon$. Observe that vertices of $\mathbb{T}$ are vertices of $L$, for every lozenge-with-diagonals tiling $L \in \mathcal{L}$. For $i=1,2$, and for $\varphi \in C_{c, 0}^{\infty}\left(\mathbb{R}^{2}\right)$ define:

$$
H_{i}^{\varepsilon} \varphi=\varepsilon^{2} \sum_{v \in V\left(\mathbb{T}^{\varepsilon}\right)} \frac{\sqrt{3}}{2} \varphi(v) h_{i}^{\varepsilon}(v) .
$$

Corollary 2. Under the joint law, the two height functions $H_{1}^{\varepsilon}, H_{2}^{\varepsilon}$ converge weakly in distribution to two independent Gaussian free fields $F_{1}$ and $F_{2}$.

\subsection{Outline of the paper}

- Section 2: statement of the explicit expressions of [3] for the Gibbs measures $\mu$ and $\mathbb{P}$, that are used in the proof of Theorem 1 and Corollary 2.

- Section 3: definition of the height function on vertices of 2-tilings of isoradial graphs.

- Section 4: definition of the Gaussian free field in the plane.

- Section 5 and Section 6: proof of Theorem 1 and Corollary 2.

\section{Minimal free energy Gibbs measure for isoradial dimer models}

In the whole of this section, we let $G$ be an isoradial graph, whose dual graph $G^{*}$ is bipartite; $B$ denotes the set of black vertices, and $W$ the set of white ones. In the proof of Theorem 1, we use the explicit expression of [3] for the minimal free energy per fundamental domain Gibbs measure $\mu$ on 2-tilings of $G$, and in the proof of Corollary 2 , we use the explicit expression of [3] for the Gibbs measure $\mathbb{P}$ on triangular quadri-tilings. The goal of this section is to state the expressions for $\mu$ and $\mathbb{P}$. In order to do so, we first define the critical weight function and the Dirac operator, introduced in [13].

\subsection{Critical weight function}

\subsubsection{Definition}

The following definition is taken from [13]. To each edge $e$ of $G^{*}$, we associate a unit side-length rhombus $R(e)$ whose vertices are the vertices of $e$ and of its dual edge $e^{*}\left(R(e)\right.$ may be degenerate). Let $\widetilde{R}=\bigcup_{e \in G^{*}} R(e)$. The critical weight function $v$ at the edge $e$ is defined by $v(e)=2 \sin \theta$, where $2 \theta$ is the angle of the rhombus $R(e)$ at the vertex it has in common with $e ; \theta$ is called the rhombus angle of the edge $e$. Note that $v(e)$ is the length of $e^{*}$.

\subsubsection{Example: critical weights for triangular quadri-tilings}

Recall that triangular quadri-tilings of $\mathcal{Q}$ correspond to two superimposed isoradial dimer models, the first on lozenge-with-diagonals tilings and the second on the equilateral triangular lattice $\mathbb{T}$. Let us note that the dual graphs of lozenge-with-diagonals tilings and of $\mathbb{T}$ are bipartite. We now compute the critical weights in the above two cases.

Consider the equilateral triangular lattice $\mathbb{T}$, then edges of its dual graph $\mathbb{T}^{*}$, known as the honeycomb lattice, all have the same rhombus angle, equal to $\pi / 3$, and the same critical weight, equal to $\sqrt{3}$.

Consider a lozenge-with-diagonals tiling $L \in \mathcal{L}$. Observe that the circumcenters of the faces of $L$ are on the boundary of the faces, so that in the isoradial embedding of the dual graph $L^{*}$ some edges have length 0 , and the rhombi associated to these edges are degenerate, see Fig. 2. Since edges of $L^{*}$ correspond to quadri-tiles of $L$, we classify them as being of type I, II, III, IV. Fig. 2 below gives the rhombus angles and the critical weights associated to edges of type I, II, III and IV, denoted by $e_{\mathrm{I}}, e_{\mathrm{II}}, e_{\mathrm{III}}, e_{\mathrm{IV}}$ respectively. 

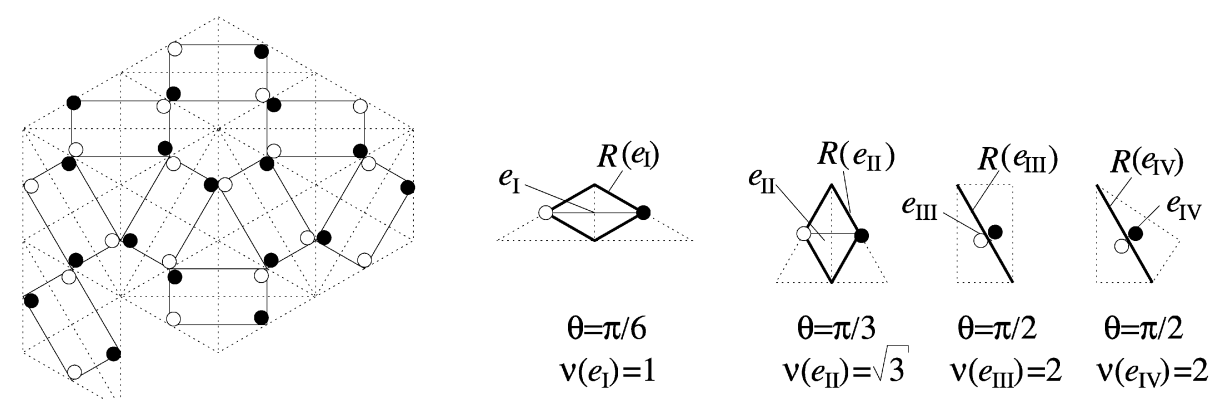

Fig. 2. Dual graph of a lozenge-with-diagonals tiling (left). Critical weights for quadri-tiles (right).

\subsection{Dirac and inverse Dirac operator}

Results in this section are due to Kenyon [13], see also Mercat [16]. Define the Hermitian matrix $K$ indexed by vertices of $G^{*}$ as follows. If $v_{1}$ and $v_{2}$ are not adjacent, $K\left(v_{1}, v_{2}\right)=0$. If $w \in W$ and $b \in B$ are adjacent vertices, then $K(w, b)=\overline{K(b, w)}$ is the complex number of modulus $v(w b)$ and direction pointing from $w$ to $b$. Another useful way to say this is as follows. Let $R(w b)$ be the rhombus associated to the edge $w b$, and denote by $w, x, b, y$ its vertices in counterclockwise order, then $K(w, b)$ is $i$ times the complex vector $x-y$. If $w$ and $b$ have the same image in the plane, then $|K(w, b)|=2$, and the direction of $K(w, b)$ is that which is perpendicular to the corresponding dual edge, and has sign determined by the local orientation. The infinite matrix $K$ defines the Dirac operator $K: \mathbb{C}^{V\left(G^{*}\right)} \rightarrow \mathbb{C}^{V\left(G^{*}\right)}$, by

$$
(K f)(v)=\sum_{u \in V\left(G^{*}\right)} K(v, u) f(u)
$$

where $V\left(G^{*}\right)$ denotes the set of vertices of the graph $G^{*}$.

The inverse Dirac operator $K^{-1}$ is defined to be the operator which satisfies

1. $K K^{-1}=\mathrm{Id}$,

2. $K^{-1}(b, w) \rightarrow 0$, when $|b-w| \rightarrow \infty$.

In [13], Kenyon proves uniqueness of $K^{-1}$, and existence by giving an explicit expression for $K^{-1}(b, w)$ as a function of the local geometry of the graph.

\subsection{Minimal free energy Gibbs measure for isoradial graphs}

If $e_{1}=w_{1} b_{1}, \ldots, e_{k}=w_{k} b_{k}$ is a subset of edges of $G^{*}$, define the cylinder set $\left\{e_{1}, \ldots, e_{k}\right\}$ of $G^{*}$ to be the set of dimer configurations of $G^{*}$ which contain the edges $e_{1}, \ldots, e_{k}$. Let $\mathcal{A}$ be the field consisting of the empty set and of the finite disjoint unions of cylinders. Denote by $\sigma(\mathcal{A})$ the $\sigma$-field generated by $\mathcal{A}$.

Theorem 3. [3] Assume $G^{*}$ is doubly periodic. Then, there is a probability measure $\mu$ on $\left(\mathcal{M}\left(G^{*}\right), \sigma(\mathcal{A})\right)$ such that for every cylinder $\left\{e_{1}, \ldots, e_{k}\right\}$ of $G^{*}$,

$$
\mu\left(e_{1}, \ldots, e_{k}\right)=\left(\prod_{i=1}^{k} K\left(w_{i}, b_{i}\right)\right) \underset{1 \leqslant i, j \leqslant k}{\operatorname{det}}\left(K^{-1}\left(b_{i}, w_{j}\right)\right) .
$$

Moreover $\mu$ is a Gibbs measure on $\mathcal{M}\left(G^{*}\right)$, and it is the unique Gibbs measure which has minimal free energy per fundamental domain among the two-parameter family of translation invariant, ergodic Gibbs measures of [17]. 


\section{Remark 4.}

- Refer to [15] for the definition of the free energy per fundamental domain.

- In [3], we prove that the periodicity assumption can be released in the case of lozenge-with-diagonals tilings. That is, given any lozenge-with-diagonals tiling of $\mathcal{L}$, Eq. (1) defines a Gibbs measure on dimer configurations of its dual graph. Although fundamental domains make no sense in case of non-periodic graphs, the minimal free energy property can still be interpreted in some wider sense.

\subsection{Gibbs measure on triangular quadri-tilings}

The construction of this section is taken from [3]. Consider the set $\mathcal{Q}$ of all triangular quadri-tilings of the plane up to isometry, and assume that quadri-tiles are assigned a positive weight function. Then the notion of Gibbs measure on $\mathcal{Q}$ is a natural extension of the one used in the case of dimer configurations of fixed graphs. It is a probability measure that satisfies the following: if a triangular quadri-tiling is fixed in an annular region, then triangular quadri-tilings inside and outside of the annulus are independent; moreover, the probability of any interior triangular quadri-tiling is proportional to the product of the weights of the quadri-tiles. Denoting by $\mathcal{M}$ the set of dimer configurations corresponding to triangular quadri-tilings of $\mathcal{Q}$, and using the bijection between $\mathcal{Q}$ and $\mathcal{M}$, we obtain the definition of a Gibbs measure on $\mathcal{M}$.

Define $\mathcal{L}^{*}$ to be set of dual graphs $L^{*}$ of lozenge-with-diagonals tilings $L \in \mathcal{L}$. Although some edges of $\mathcal{L}^{*}$ have length 0 , we think of them as edges of the one skeleton of the graphs, so that to every edge of $\mathcal{L}^{*}$, there corresponds a unique quadri-tile. Let $e$ be an edge of $\mathcal{L}^{*}$, and let $q_{e}$ be the corresponding quadri-tile, then $q_{e}$ is made of two adjacent right triangles. If the two triangles share the hypotenuse edge, they belong to two adjacent lozenges; else if they share a leg, they belong to the same lozenge. Let us call these lozenge(s) the lozenge(s) associated to the edge $e$, and denote it/them by $\mathrm{I}_{e}$ (that is $\mathrm{I}_{e}$ consists of either one or two lozenges). Let $\mathrm{k}_{e}$ be the edge(s) of $\mathbb{T}^{*}$ corresponding to the lozenge(s) $\left.\right|_{e}$. Let us introduce one more definition, if $\left\{e_{1}, \ldots, e_{k}\right\}$ is a subset of edges of $\mathcal{L}^{*}$, then the cylinder set $\left\{e_{1}, \ldots, e_{k}\right\}$ is the set of dimer configurations of $\mathcal{M}$ which contain these edges. Denote by $\mathcal{C}$ the field consisting of the empty set and of the finite disjoint unions of cylinders. Denote by $\sigma(\mathcal{C})$ the $\sigma$-field generated by $\mathcal{C}$.

Consider a lozenge-with-diagonals tiling $L \in \mathcal{L}$, and denote by $\mu^{L}$ the minimal free energy per fundamental domain Gibbs measure on $\left(\mathcal{M}\left(L^{*}\right), \sigma(\mathcal{A})\right)$ given by Theorem 3, where $\sigma(\mathcal{A})$ is the $\sigma$-field of cylinders of $\mathcal{M}\left(L^{*}\right)$. Similarly, denote by $\mu^{\mathbb{T}}$ the minimal free energy per fundamental domain Gibbs measure on $\left(\mathcal{M}\left(\mathbb{T}^{*}\right), \sigma(\mathcal{B})\right)$, where $\sigma(\mathcal{B})$ is the $\sigma$-field of cylinders of $\mathcal{M}\left(\mathbb{T}^{*}\right)$.

Let us define $\tilde{\mu}^{L}$ on $(\mathcal{M}, \sigma(\mathcal{C}))$ by:

$$
\tilde{\mu}^{L}\left(e_{1}, \ldots, e_{k}\right)= \begin{cases}\mu^{L}\left(e_{1}, \ldots, e_{k}\right) & \text { if the lozenges } \mathrm{I}_{e_{1}}, \ldots, \mathrm{l}_{e_{k}} \text { belong to } \mathrm{L}, \\ 0 & \text { else, }\end{cases}
$$

where we recall that $L$ is the lozenge tiling obtained from the lozenge-with-diagonals tiling $L$ by removing the diagonals. Then, it is easy to check that $\tilde{\mu}^{L}$ is a probability measure on $(\mathcal{M}, \sigma(\mathcal{C}))$. In order to simplify notations, we write $\mu^{L}$ for $\tilde{\mu}^{L}$ whenever no confusion occurs.

Now, on $\left(\mathcal{M} \times \mathcal{M}\left(\mathbb{T}^{*}\right), \mathcal{B} \times \mathcal{C}\right)$, define:

$$
\mathbb{P}\left(\left(e_{1}, \ldots, e_{k}\right) \times\left(k_{1}, \ldots, k_{m}\right)\right)=\sum_{\left\{\mathrm{L}^{*} \in \mathcal{M}\left(\mathbb{T}^{*}\right): k_{1}, \ldots, k_{m} \in \mathrm{L}^{*}\right\}} \mu^{L}\left(e_{1}, \ldots, e_{k}\right) \mathrm{d} \mu^{\mathbb{T}}\left(\mathrm{L}^{*}\right) .
$$

Using Kolmogorov's extension theorem, $\mathbb{P}$ extends to a probability measure on $\left(\mathcal{M} \times \mathbb{T}^{*}, \sigma(\mathcal{B} \times \mathcal{C})\right)$. Let us also denote by $\mathbb{P}$ the marginal of $\mathbb{P}$ on $\mathcal{M}$, then in [3], $\mathbb{P}$ is shown to be a Gibbs measure on $\mathcal{M}$, and conjectured to be of minimal free energy per fundamental domain among a four-parameter family of translation invariant, ergodic Gibbs measures.

\section{Height functions}

In the whole of this section, we let $G$ be an isoradial graph whose dual graph $G^{*}$ is bipartite; as before, $B$ denotes the set of black vertices, $W$ the set of white ones. We define the height function $h$ on vertices of 2-tilings of $G$, whose fluctuations are described in Theorem 1. As in [15], see also [2], $h$ is defined using flows. 
The bipartite coloring of the vertices of $G^{*}$ induces an orientation of the faces of $G$ : color the dual faces of the black (white) vertices black (white); orient the boundary edges of the black faces counterclockwise, the boundary edges of the white faces are then oriented clockwise.

\subsection{Definition}

Let us first define a flow $\omega_{0}$ on the edges of $G^{*}$. Consider an edge $w b$ of $G^{*}$, then $R(w b)$ is the rhombus associated to $w b$, and $\theta_{w b}$ is the corresponding rhombus angle. Define $\omega_{0}$ to be the white-to-black flow, which flows by $\theta_{w b} / \pi$ along every edge $w b$ of $G^{*}$.

Lemma 5. The flow $\omega_{0}$ has divergence 1 at every white vertex, and -1 at every black vertex of $G^{*}$.

Proof. By definition of the rhombus angle, we have

$$
\forall w \in W, \quad \sum_{b: b \sim w} 2 \theta_{w b}=2 \pi ; \quad \forall b \in B, \quad \sum_{w: w \sim b} 2 \theta_{w b}=2 \pi .
$$

Now, consider a 2-tiling $T$ of $G$, and let $M$ be the corresponding perfect matching of $G^{*}$. Then $M$ defines a white-toblack unit flow $\omega$ on the edges of $G^{*}$ : flow by 1 along every edge of $M$, from the white vertex to the black one. The difference $\omega_{0}-\omega$ is a divergence free flow, which means that the quantity of flow that enters any vertex of $G^{*}$ equals the quantity of flow which exits that same vertex.

We are ready for the definition of the height function $h$. Choose a vertex $v_{0}$ of $G$, and fix $h\left(v_{0}\right)=0$. For every other vertex $v$ of $T$, take an edge-path $\gamma$ of $G$ from $v_{0}$ to $v$. If an edge $u v$ of $\gamma$ is oriented in the direction of the path, and if we denote by $e$ its dual edge, then $h$ increases by $\omega_{0}(e)-\omega(e)$ along $u v$; if an edge $u v$ is oriented in the opposite direction, then $h$ decreases by the same quantity along $u v$. As a consequence of the fact that $\omega_{0}-\omega$ is a divergence free flow, the height function $h$ is well defined.

The following lemma gives a correspondence between height functions defined on vertices of $G$, and 2-tilings of $G$.

\section{Lemma 6. Let $\tilde{h}$ be an $\mathbb{R}$-valued function on vertices of $G$ satisfying}

- $\tilde{h}\left(v_{0}\right)=0$,

- $\tilde{h}(v)-\tilde{h}(u)=\omega_{0}(e)$ or $\omega_{0}(e)-1$ for any edge uv oriented from $u$ to $v$, where e denotes the dual edge of $u v$.

Then, there is a bijection between functions $\tilde{h}$ satisfying these two conditions, and 2-tilings of $G$.

Proof. The idea of the proof closely follows [5]. Let $T$ be a 2-tiling of $G, M$ be the corresponding matching, and $\omega$ be the unit white-to-black flow defined by $M$. Then, the height function $h$ satisfies the conditions of the lemma: consider an edge $u v$ of $G$ oriented from $u$ to $v$ and denote by $e$ its dual edge, then $h(v)-h(u)=\omega_{0}(e)-\omega(e)$, and by definition $\omega(e)=0$ or 1 .

Conversely, consider an $\mathbb{R}$-valued function $\tilde{h}$ as in the lemma. Let us construct a 2-tiling $T$ whose height function is $\tilde{h}$. Consider a black face $F$ of $G$, and let $e_{1}, \ldots, e_{m}$ be the dual edges of its boundary edges. Then $\sum_{i=1}^{m} \omega_{0}\left(e_{i}\right)=1$, so that there is exactly one boundary edge $u v$ along which $\tilde{h}(v)-\tilde{h}(u)$ is $\omega_{0}\left(e_{i}\right)-1$ (where $e_{i}$ is the dual edge of $u v$ ). To the face $F$, we associate the 2-tile of $G$ which is crossed by the edge $u v$. Repeating this procedure for all black faces of $G$, we obtain $T$.

\subsection{Interpretation}

The discrete interface interpretation of 2-tilings was first given by Thurston in the case of lozenges [19]. Following him, a 2-tiling of $G$ can be seen as a discrete 2-dimensional surface $S$ in a 3-dimensional space that has been projected orthogonally to the plane; the "height" of $S$ is given by the function $h$. Stated in physics terminology, 2-tilings of $G$ are random interfaces in dimension $2+1$. 


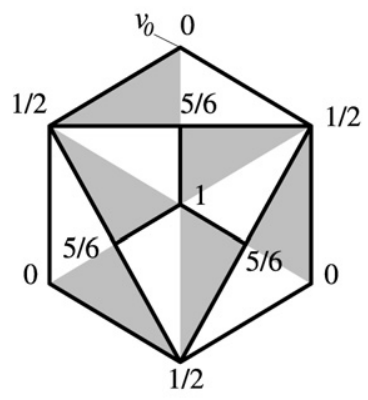

$T$

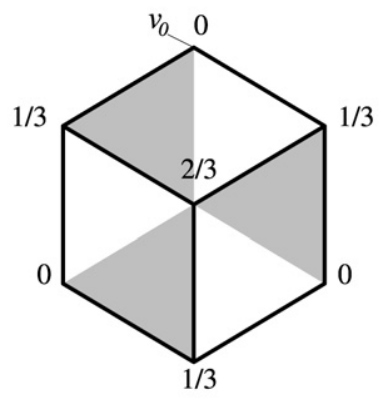

$\mathrm{L}(T)$

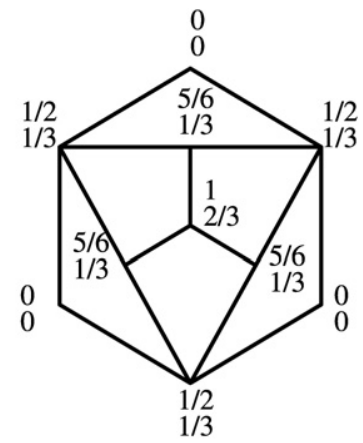

$T$

Fig. 3. From left to right: triangular quadri-tiling T and first height function $h_{1}$, underlying lozenge tiling $\mathrm{L}(T)$ and second height function $h_{2}$, T with height functions $h_{1}$ (above) and $h_{2}$ (below).

\subsection{Example: the two height functions of triangular quadri-tilings}

Consider a triangular quadri-tiling $T \in \mathcal{Q}$. Then, recall that $h_{1}$ assigns to vertices of $T$ the "height" of $T$ interpreted as a 2-tiling of its underlying lozenge-with-diagonals tiling $L(T)$, and $h_{2}$ assigns to vertices of $T$ the "height" of $\mathrm{L}(T)$ interpreted as a 2-tiling of $\mathbb{T}$. Let us now explicitly compute $h_{1}$ and $h_{2}$.

The definition of the flow $\omega_{0}(L(T))$ on edges of $L(T)^{*}$ uses the rhombus angles of the edges of $L(T)^{*}$. These have been computed in Section 2.1.2 and are equal to $\frac{\pi}{6}, \frac{\pi}{3}, \frac{\pi}{2}$ for edges of type I, II, III and IV respectively. Hence if $u v$ is the boundary edge of a quadri-tile of $T$, oriented from $u$ to $v$, then the height change of $h_{1}$ along $u v$ is $\frac{1}{6}, \frac{1}{3}, \frac{1}{2}$ depending on whether the dual edge $e$ of the edge $u v$ is of type I, II, III or IV respectively, see Fig. 3.

In a similar way, the flow $\omega_{0}\left(\mathbb{T}^{*}\right)$ on edges of $\mathbb{T}^{*}$ flows by $\frac{1}{3}$ along every edge, so that if $u^{\prime} v^{\prime}$ be a boundary edge of a lozenge of $\mathrm{L}(T)$ oriented from $u^{\prime}$ to $v^{\prime}$, then the height change of $h_{2}$ along $u^{\prime} v^{\prime}$ is equal to $\frac{1}{3}$. Thinking of a lozenge as the projection to the plane of the face of a cube [19], there is a natural way to assign a value for $h_{2}$ at the vertex at the crossing of the diagonals of the lozenges, see Fig. 3.

By means of $h_{1}$ and $h_{2}$, a triangular quadri-tiling $T$ of $\mathcal{Q}$ is interpreted in [3] as a 2-dimensional discrete surface $S_{1}$ in a 4-dimensional space that has been projected orthogonally to the plane. $S_{1}$ can also be projected to $\frac{1}{3} \widetilde{\mathbb{Z}}^{3}\left(\widetilde{\mathbb{Z}}^{3}\right.$ is the space $\mathbb{Z}^{3}$ where the cubes are drawn with diagonals on their faces), and one obtains a surface $S_{2}$. When projected to the plane $S_{2}$ is the underlying lozenge-with-diagonals tiling $L(T)$. This can be restated by saying that triangular quadri-tilings of $\mathcal{Q}$ are discrete interfaces in dimension $2+2$.

\section{Gaussian free field in the plane}

Theorem 1 and Corollary 2 prove convergence of the height function $h$ to a Gaussian free field. The goal of this section is to define the Gaussian free field in the plane. We refer to $[6,18]$ for other ways of defining the Gaussian free field.

\subsection{Green function of the plane, and Dirichlet energy}

The Green function of the plane, denoted by $g$, is the kernel of the Laplace equation in the plane, it satisfies $\Delta_{x} g(x, y)=\delta_{x}(y)$, where $\delta_{x}$ is the Dirac distribution at $x$. Up to an additive constant, $g$ is given by

$$
g(x, y)=-\frac{1}{2 \pi} \log |x-y| \text {. }
$$

Define the following bilinear form

$$
\begin{aligned}
G: C_{c, 0}^{\infty}\left(\mathbb{R}^{2}\right) \times C_{c, 0}^{\infty}\left(\mathbb{R}^{2}\right) & \longrightarrow \mathbb{R}, \\
\left(\varphi_{1}, \varphi_{2}\right) & \longmapsto G\left(\varphi_{1}, \varphi_{2}\right)=\int_{\mathbb{R}^{2}} \int_{\mathbb{R}^{2}} g(x, y) \varphi_{1}(x) \varphi_{2}(y) \mathrm{d} x \mathrm{~d} y .
\end{aligned}
$$


$G(\varphi, \varphi)$ is called the Dirichlet energy of $\varphi$. Let us consider the topology induced by the $L^{\infty}$ norm on $C_{c, 0}^{\infty}\left(\mathbb{R}^{2}\right)$. Then, $G$ is a continuous, positive definite, bilinear form.

\subsection{Random distributions}

The following definitions are taken from [7]. A random function $F$ associates to every function $\varphi \in C_{c, 0}^{\infty}\left(\mathbb{R}^{2}\right)$ a real random variable $F \varphi$. For $\varphi_{1}, \ldots, \varphi_{k} \in C_{c, 0}^{\infty}\left(\mathbb{R}^{2}\right)$, we suppose that the joint probabilities $a_{n} \leqslant F \varphi_{n}<b_{n}, 1 \leqslant n \leqslant k$ are given, and we ask that they satisfy the compatibility relation. A random function $F$ is linear if $\forall \varphi_{1}, \varphi_{2} \in C_{c, 0}^{\infty}\left(\mathbb{R}^{2}\right)$,

$$
F\left(\alpha \varphi_{1}+\beta \varphi_{2}\right)=\alpha F \varphi_{1}+\beta F \varphi_{2} .
$$

It is continuous if convergence of the functions $\varphi_{n_{j}}$ to $\varphi_{j}(1 \leqslant j \leqslant k)$ implies

$$
\lim _{n \rightarrow \infty}\left(F \varphi_{n_{1}}, \ldots, F \varphi_{n_{k}}\right)=\left(F \varphi_{1}, \ldots, F \varphi_{k}\right),
$$

that is, if $P(x)$ (resp. $\left.P_{n}(x)\right)$ is the probability measure corresponding to the random variable $\left(F \varphi_{1}, \ldots, F \varphi_{k}\right)$ (resp. $\left.\left(F \varphi_{n_{1}}, \ldots, F \varphi_{n_{k}}\right)\right)$, then for any bounded continuous function $f$

$$
\lim _{n \rightarrow \infty} \int f\left(x_{1}, \ldots, x_{k}\right) \mathrm{d} P_{n}(x)=\int f\left(x_{1}, \ldots, x_{k}\right) \mathrm{d} P(x) .
$$

A random distribution $F$ is a random function which is linear and continuous. It is said to be Gaussian if for every linearly independent functions $\varphi_{1}, \ldots, \varphi_{k} \in C_{c, 0}^{\infty}\left(\mathbb{R}^{2}\right)$, the random vector $\left(F \varphi_{1}, \ldots, F \varphi_{k}\right)$ is Gaussian.

Two random distributions $F$ and $G$ are said to be independent if for any functions $\varphi_{1}, \ldots, \varphi_{k} \in C_{c, 0}^{\infty}\left(\mathbb{R}^{2}\right)$, the random vectors $\left(F \varphi_{1}, \ldots, F \varphi_{k}\right)$ and $\left(G \varphi_{1}, \ldots, G \varphi_{k}\right)$ are independent.

\subsection{Gaussian free field in the plane}

Lemma 7. [1] If $G: C_{c, 0}^{\infty}\left(\mathbb{R}^{2}\right) \times C_{c, 0}^{\infty}\left(\mathbb{R}^{2}\right) \rightarrow \mathbb{R}$ is a bilinear, continuous, positive definite form, then there exists a Gaussian random distribution $F$, whose covariance function is given by

$$
\mathbb{E}\left(F \varphi_{1} F \varphi_{2}\right)=G\left(\varphi_{1}, \varphi_{2}\right) .
$$

Using Lemma 7, we define a Gaussian free field in the plane to be a Gaussian random distribution whose covariance function is

$$
\mathbb{E}\left(F \varphi_{1} F \varphi_{2}\right)=-\frac{1}{2 \pi} \int_{\mathbb{R}^{2}} \int_{\mathbb{R}^{2}} \log |x-y| \varphi_{1}(x) \varphi_{2}(y) \mathrm{d} x \mathrm{~d} y .
$$

\section{Proof of Theorem 1}

We place ourselves in the context of Theorem 1: $G$ is an isoradial graph whose dual graph $G^{*}$ is doubly periodic and bipartite; edges of $G^{*}$ are assigned the critical weight function, and $G^{\varepsilon}$ is the graph $G$ whose edge-lengths have been multiplied by $\varepsilon$. Recall the following notations: $H^{\varepsilon} \varphi=\varepsilon^{2} \sum_{v \in V\left(G^{\varepsilon}\right)} a\left(v^{*}\right) \varphi(v) h^{\varepsilon}(v), \mu$ is the minimal free energy per fundamental domain Gibbs measure for the dimer model on $G^{*}$ of Section 2.3, and $F$ is a Gaussian free field in the plane.

Since the random vector $\left(F \varphi_{1}, \ldots, F \varphi_{k}\right)$ is Gaussian, to prove convergence of $\left(H^{\varepsilon} \varphi_{1}, \ldots, H^{\varepsilon} \varphi_{k}\right)$ to $\left(F \varphi_{1}, \ldots\right.$, $\left.F \varphi_{k}\right)$, it suffices to prove convergence of the moments of $\left(H^{\varepsilon} \varphi_{1}, \ldots, H^{\varepsilon} \varphi_{k}\right)$ to those of $\left(F \varphi_{1}, \ldots, F \varphi_{k}\right)$; that is we need to show that for every $k$-tuple of positive integers $\left(m_{1}, \ldots, m_{k}\right)$, we have

$$
\lim _{\varepsilon \rightarrow 0} \mathbb{E}\left[\left(H^{\varepsilon} \varphi_{1}\right)^{m_{1}} \ldots\left(H^{\varepsilon} \varphi_{k}\right)^{m_{k}}\right]=\mathbb{E}\left[\left(F \varphi_{1}\right)^{m_{1}} \ldots\left(F \varphi_{k}\right)^{m_{k}}\right]
$$

In Section 5.1, we prove two properties of the height function $h$, and in Section 5.2, we give the asymptotic formula of [13] for the inverse Dirac operator $K^{-1}$. Using these results in Section 5.3, we prove a formula for the limit (as $\varepsilon \rightarrow 0$ ) of the $k$ th moment of $h$. This allows us to show convergence of $\mathbb{E}\left[\left(H^{\varepsilon} \varphi\right)^{k}\right]$ to $\mathbb{E}\left[(F \varphi)^{k}\right]$ in Section 5.4. One then obtains Eq. (2) by choosing $\varphi$ to be a suitable linear combination of the $\varphi_{i}$ 's.

As before, $B$ denotes the set of black vertices of $G^{*}$, and $W$ the set of white ones. Moreover, we suppose that faces of $G$ have the orientation induced by the bipartite coloring of the vertices of $G^{*}$. 


\subsection{Properties of the height function}

Let $u, v$ be two vertices of $G$, and let $\gamma$ be an edge-path of $G$ from $u$ to $v$. First, consider edges of $\gamma$ which are oriented in the direction of the path, that is edges which have a black face of $G$ on the left, and denote by $f_{1}, \ldots, f_{n}$ their dual edges. Hence an edge $f_{j}$ consists of a black vertex on the left of $\gamma$, and of a white one on the right. Similarly, consider edges of $\gamma$ which are oriented in the opposite direction, and denote by $e_{1}, \ldots, e_{m}$ their dual edges, hence an edge $e_{i}$ consists of a white vertex on the left of $\gamma$, and of a black one on the right. Let $\mathbb{I}_{e}$ be the indicator function of $\mathcal{M}\left(G^{*}\right): \mathbb{I}_{e}(M)=1$, if the edge $e$ belongs to the dimer configuration $M$ of $G^{*}$, and 0 otherwise.

\section{Lemma 8.}

$$
h(v)-h(u)=\sum_{j=1}^{m}\left(\mathbb{I}_{e_{j}}-\mu\left(e_{j}\right)\right)+\sum_{j=1}^{n}\left(-\mathbb{I}_{f_{j}}+\mu\left(f_{j}\right)\right)
$$

Proof. Let $e_{j}$ be the dual edge of an edge $u_{j} v_{j}$ of $\gamma$ oriented from $v_{j}$ to $u_{j}$. Denote by $\theta_{j}$ the rhombus angle of the edge $e_{j}$, then by Lemma 6 ,

$$
h\left(v_{j}\right)-h\left(u_{j}\right)= \begin{cases}-\frac{\theta_{j}}{\pi}+1 & \text { if the edge } e_{j} \text { belongs to the dimer configuration of } G^{*}, \\ -\frac{\theta_{j}}{\pi} & \text { else. }\end{cases}
$$

Hence

$$
h\left(v_{j}\right)-h\left(u_{j}\right)=\left(-\frac{\theta_{j}}{\pi}+1\right) \mathbb{I}_{e_{j}}-\frac{\theta_{j}}{\pi}\left(1-\mathbb{I}_{e_{j}}\right)=\mathbb{I}_{e_{j}}-\frac{\theta_{j}}{\pi} .
$$

Moreover, a direct computation using formula (1) yields $\mu\left(e_{j}\right)=\theta_{j} / \pi$, so

$$
h\left(v_{j}\right)-h\left(u_{j}\right)=\mathbb{I}_{e_{j}}-\mu\left(e_{j}\right) .
$$

Similarly, when $f_{j}$ is the dual edge of an edge $u_{j}^{\prime} v_{j}^{\prime}$ of $\gamma$ oriented from $u_{j}^{\prime}$ to $v_{j}^{\prime}$, we obtain $h\left(v_{j}^{\prime}\right)-h\left(u_{j}^{\prime}\right)=-\mathbb{I}_{f_{j}}+$ $\mu\left(f_{j}\right)$, and we conclude

$$
h(v)-h(u)=\sum_{j=1}^{m} h\left(v_{j}\right)-h\left(u_{j}\right)+\sum_{j=1}^{n} h\left(v_{j}^{\prime}\right)-h\left(u_{j}^{\prime}\right)=\sum_{j=1}^{m}\left(\mathbb{I}_{e_{j}}-\mu\left(e_{j}\right)\right)+\sum_{j=1}^{n}\left(-\mathbb{I}_{f_{j}}+\mu\left(f_{j}\right)\right) .
$$

\section{Lemma 9.}

$$
\mathbb{E}_{\mu}[h(v)-h(u)]=0 .
$$

Proof. By Lemma 8 we have,

$$
\mathbb{E}_{\mu}[h(v)-h(u)]=\sum_{j=1}^{m} \mathbb{E}_{\mu}\left[\mathbb{I}_{e_{j}}-\mu\left(e_{j}\right)\right]+\sum_{j=1}^{n} \mathbb{E}_{\mu}\left[-\mathbb{I}_{f_{j}}+\mu\left(f_{j}\right)\right]=0 .
$$

\subsection{Asymptotics of the inverse Dirac operator $K^{-1}$}

In order to state the asymptotic formula of [13] for the inverse of the Dirac operator $K$ indexed by vertices of $G^{*}$, we let $w \in W$ be a white vertex of $G^{*}, x$ any other vertex of $G^{*}$, and define the rational function $f_{w x}(z)$ of [13]. Recall that $\widetilde{R}$ is the set of rhombi associated to edges of $G^{*}$, and consider $w=x_{0}, x_{1}, x_{2}, \ldots, x_{k}=x$ an edge-path of $\widetilde{R}$ from $w$ to $x$. Each edge $x_{j} x_{j+1}$ has exactly one vertex of $G^{*}$ (the other is a vertex of $G$ ). Direct the edge away from this vertex if it is white, and towards this vertex if it is black. Let $\mathrm{e}^{\mathrm{i} \alpha_{j}}$ be the corresponding vector in $\widetilde{R}$ (which may point contrary to the direction of the path). Then, $f_{w x}$ is defined inductively along the path, starting from

$$
f_{w w}(z)=1 \text {. }
$$


If the edge leads away from a white vertex, or towards a black vertex, then

$$
f_{w x_{j+1}}(z)=\frac{f_{w x_{j}}(z)}{1-\mathrm{e}^{\mathrm{i} \alpha_{j}}},
$$

else, if it leads towards a white vertex, or away from a black vertex, then

$$
f_{w x_{j+1}}(z)=f_{w x_{j}}(z) \cdot\left(z-\mathrm{e}^{\mathrm{i} \alpha_{j}}\right) .
$$

The function $f_{w x}(z)$ is well defined (i.e. independent of the edge-path of $\widetilde{R}$ from $w$ to $x$ ). Then, Kenyon gives the following asymptotics for the inverse Dirac operator $K^{-1}$ :

Theorem 10. [13] Asymptotically, as $|b-w| \rightarrow \infty$,

$$
K^{-1}(b, w)=\frac{1}{2 \pi}\left(\frac{1}{b-w}+\frac{f_{w b}(0)}{\bar{b}-\bar{w}}\right)+\mathrm{O}\left(\frac{1}{|b-w|^{2}}\right) .
$$

\subsection{Moment formula}

Let $u_{1}, \ldots, u_{k}, v_{1}, \ldots, v_{k}$ be distinct points of $\mathbb{R}^{2}$, and let $\gamma_{1}, \ldots, \gamma_{k}$ be pairwise disjoint, simple, smooth paths such that $\gamma_{j}$ runs from $u_{j}$ to $v_{j}$. Let $u_{j}^{\varepsilon}$, $v_{j}^{\varepsilon}$ be vertices of $G^{\varepsilon}$ lying within $\mathrm{O}(\varepsilon)$ of $u_{j}$ and $v_{j}$ respectively. In order to simplify notations, we write $h$ for the unnormalized height function $h^{\varepsilon}$ on 2-tilings of $G^{\varepsilon}$. Then, we have

Proposition 11. For every $k \in \mathbb{N}, k \geqslant 2$

$$
\lim _{\varepsilon \rightarrow 0} \mathbb{E}\left[\left(h\left(v_{1}^{\varepsilon}\right)-h\left(u_{1}^{\varepsilon}\right)\right) \cdots\left(h\left(v_{k}^{\varepsilon}\right)-h\left(u_{k}^{\varepsilon}\right)\right)\right]=\frac{(-i)^{k}}{(2 \pi)^{k}} \sum_{\rho=0,1}(-1)^{k \rho}\left(\int_{\gamma_{1}} \ldots \int_{\substack { \gamma_{k} \\
\begin{subarray}{c}{i, j \in[1, k] \\
i \neq j{ \gamma _ { k } \\
\begin{subarray} { c } { i , j \in [ 1 , k ] \\
i \neq j } }\end{subarray}}\left(\frac{1}{z_{i}^{\rho}-z_{j}^{\rho}}\right) \mathrm{d} z_{1}^{\rho} \cdots d z_{k}^{\rho}\right),
$$

where $z_{i}^{0}=z_{i}$ and $z_{i}^{1}=\bar{z}_{i}$.

Proof. The structure of the proof of Proposition 11 is essentially that of [11], but since we work in a much more general setting, each step is adapted in a non-trivial way. The proof being quite long, here is a short outline: after having initiated the proof, we derive and show four lemmas (Lemmas 12-15); then, after the last of the four lemmas has been shown, we conclude the proof of Proposition 11.

Let $\gamma_{1}^{\varepsilon}, \ldots, \gamma_{k}^{\varepsilon}$ be pairwise disjoint paths of $G^{\varepsilon}$, such that $\gamma_{j}^{\varepsilon}$ runs from $u_{j}^{\varepsilon}$ to $v_{j}^{\varepsilon}$ and approximates $\gamma_{j}$ within $\mathrm{O}(\varepsilon)$. For every $j$, denote by $f_{j s}$ the dual edge of the $s$ th edge of the path $\gamma_{j}^{\varepsilon}$, which is oriented in the direction of the path: $f_{j s}$ consists of a black vertex on the left of $\gamma_{j}^{\varepsilon}$, and of a white one on the right. Denote by $e_{j t}$ the dual edge of the $t$ th edge of the path $\gamma_{j}^{\varepsilon}$, which is oriented in the opposite direction: $e_{j t}$ consists of a black vertex on the right of $\gamma_{j}^{\varepsilon}$, and of a white one on the left. Using Lemma 8, we obtain

$$
\begin{aligned}
\mathbb{E} & {\left[\left(h\left(v_{1}^{\varepsilon}\right)-h\left(u_{1}^{\varepsilon}\right)\right) \cdots\left(h\left(v_{k}^{\varepsilon}\right)-h\left(u_{k}^{\varepsilon}\right)\right)\right] } \\
& =\mathbb{E}\left[\sum_{t_{1}}\left(\mathbb{I}_{e_{1 t_{1}}}-\mu\left(e_{1 t_{1}}\right)\right)-\sum_{s_{1}}\left(\mathbb{I}_{f_{1 s_{1}}}-\mu\left(f_{1 s_{1}}\right)\right)\right] \ldots\left[\sum_{t_{k}}\left(\mathbb{I}_{e_{k t_{k}}}-\mu\left(e_{k t_{k}}\right)\right)-\sum_{s_{k}}\left(\mathbb{I}_{f_{k s_{k}}}-\mu\left(f_{k s_{k}}\right)\right)\right] \\
& =\sum_{t_{1}, \ldots, t_{k}} \mathbb{E}\left[\mathbb{I}_{e_{1 t_{1}}}-\mu\left(e_{1 t_{1}}\right)\right] \cdots\left[\mathbb{I}_{e_{k t_{k}}}-\mu\left(e_{k t_{k}}\right)\right]-\cdots+(-1)^{k} \sum_{s_{1}, \ldots, s_{k}} \mathbb{E}\left[\mathbb{I}_{f_{1 s_{1}}}-\mu\left(f_{1 s_{1}}\right)\right] \cdots\left[\mathbb{I}_{f_{k s_{k}}}-\mu\left(f_{k s_{k}}\right)\right] \\
& =\sum_{\delta_{1}, \ldots, \delta_{k} \in\{0,1\}} \sum_{t_{1}^{\delta_{1}}, \ldots, t_{k}^{\delta_{k}}}(-1)^{\delta_{1}+\cdots+\delta_{k}} \mathbb{E}\left[\mathbb{I}_{e_{1 t_{1} \delta_{1}}}-\mu\left(e_{1 t_{1}^{\delta_{1}}}\right)\right] \cdots\left[\mathbb{I}_{e_{k t_{k} \delta_{k}}}-\mu\left(e_{k t_{k} \delta_{k}}\right)\right],
\end{aligned}
$$

where

$$
t_{j}^{\delta_{j}}=\left\{\begin{array}{ll}
t_{j} & \text { if } \delta_{j}=0, \\
s_{j} & \text { if } \delta_{j}=1,
\end{array} e_{j t_{j}{ }_{j}}=e^{\delta_{j}}{ }_{j t_{j}}^{\delta_{j}}= \begin{cases}e_{j t_{j}} & \text { if } \delta_{j}=0 \\
f_{j s_{j}} & \text { if } \delta_{j}=1\end{cases}\right.
$$


For the time being, let us drop the second subscript. Write $e_{j}=w_{j} b_{j}$ and $f_{j}=w_{j}^{\prime} b_{j}^{\prime}$. Moreover, let us introduce the notation $w_{j}^{\delta_{j}}$, where $w_{j}^{\delta_{j}}=w_{j}$ if $\delta_{j}=0$, and $w_{j}^{\delta_{j}}=w_{j}^{\prime}$ if $\delta_{j}=1$, similarly we introduce the notation $b_{j}^{\delta_{j}}$. Hence we can write a generic term of (4) as

$$
(-1)^{\delta_{1}+\cdots+\delta_{k}} \mathbb{E}\left[\left(\mathbb{I}_{w_{1}^{\delta_{1}} b_{1}^{\delta_{1}}}-\mu\left(w_{1}^{\delta_{1}} b_{1}^{\delta_{1}}\right)\right) \cdots\left(\mathbb{I}_{w_{k}^{\delta_{k}} b_{k}^{\delta_{k}}}-\mu\left(w_{k}^{\delta_{k}} b_{k}^{\delta_{k}}\right)\right)\right]
$$

\section{Lemma 12. [8]}

$$
\begin{aligned}
& (-1)^{\delta_{1}+\cdots+\delta_{k}} \mathbb{E}\left[\left(\mathbb{I}_{w_{1}^{\delta_{1}} b_{1}^{\delta_{1}}}-\mu\left(w_{1}^{\delta_{1}} b_{1}^{\delta_{1}}\right)\right) \cdots\left(\mathbb{I}_{w_{k}^{\delta_{k}} b_{k}^{\delta_{k}}}-\mu\left(w_{k}^{\delta_{k}} b_{k}^{\delta_{k}}\right)\right)\right]=
\end{aligned}
$$

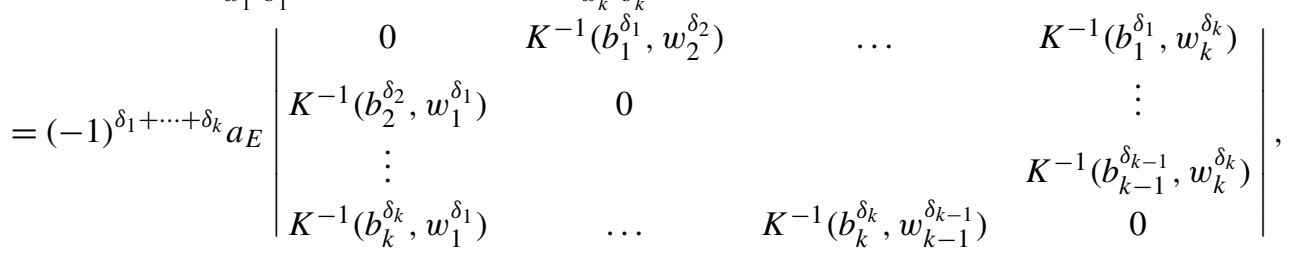

where $a_{E}=\prod_{j=1}^{k} K\left(w_{j}^{\delta_{j}}, b_{j}^{\delta_{j}}\right)$, and $K$ is the Dirac operator indexed by vertices of $G^{*}$.

A typical term in the expansion of (5) is

$$
(-1)^{\delta_{1}+\cdots+\delta_{k}} a_{E} \operatorname{sgn} \sigma K^{-1}\left(b_{1}^{\delta_{1}}, w_{\sigma(1)}^{\delta_{\sigma(1)}}\right) \cdots K^{-1}\left(b_{k}^{\delta_{k}}, w_{\sigma(k)}^{\delta_{\sigma(k)}}\right)
$$

where $\sigma \in \widetilde{S_{k}}$, and $\widetilde{S_{k}}$ is the set of permutations of $k$ elements, with no fixed points. To simplify notations, let us assume $\sigma$ is a $k$-cycle, hence (6) becomes

$$
(-1)^{\delta_{1}+\cdots+\delta_{k}} a_{E} \operatorname{sgn} \sigma K^{-1}\left(b_{1}^{\delta_{1}}, w_{2}^{\delta_{2}}\right) \cdots K^{-1}\left(b_{k}^{\delta_{k}}, w_{1}^{\delta_{1}}\right)
$$

Lemma 13. When $\varepsilon$ is small, and for every $\delta_{1}, \ldots, \delta_{k} \in\{0,1\}$,

$$
\varepsilon^{k} a_{E}=(-i)^{k}(-1)^{\delta_{1}+\cdots+\delta_{k}} \mathrm{~d} z_{1}^{\delta_{1}} \cdots \mathrm{d} z_{k}^{\delta_{k}},
$$

where $d z_{j}^{\delta_{j}}$ denotes the dual edge of the edge $w_{j}^{\delta_{j}} b_{j}^{\delta_{j}}$ (oriented in the direction of $\gamma_{j}^{\varepsilon}$ ).

Proof. Let $x_{j} y_{j}$ be an edge of the path $\gamma_{j}^{\varepsilon}$ where $x_{j}$ precedes $y_{j}$. We can write

$$
x_{j} y_{j}=\varepsilon \ell\left(x_{j} y_{j}\right) \mathrm{e}^{\mathrm{i} \theta_{j}},
$$

where $\ell\left(x_{j} y_{j}\right)$ is the length of the edge $x_{j} y_{j}$ in $G$, and $\theta_{j}$ is the direction from $x_{j}$ to $y_{j}$. Let us first consider the case of an edge $x_{j} y_{j}$ oriented in the direction of the path, that is the dual edge $w_{j}^{\prime} b_{j}^{\prime}$ of $x_{j} y_{j}$ has its black vertex on the left of $\gamma_{j}^{\varepsilon}$. By definition of the Dirac operator, we have $K\left(w_{j}^{\prime}, b_{j}^{\prime}\right)=\ell\left(x_{j} y_{j}\right) \mathrm{e}^{\mathrm{i} \theta_{j}} \mathrm{e}^{\mathrm{i} \frac{\pi}{2}}$. Next we consider the case of an edge $x_{j} y_{j}$ oriented in the opposite direction, that is its dual edge $w_{j} b_{j}$ has its black vertex on the right of $\gamma_{j}^{\varepsilon}$. Again, using the definition of the Dirac operator, we obtain $K\left(w_{j}, b_{j}\right)=\ell\left(x_{j} y_{j}\right) \mathrm{e}^{\mathrm{i} \theta_{j}} \mathrm{e}^{-\mathrm{i} \frac{\pi}{2}}$. We summarize the two cases by the following equation

$$
K\left(w_{j}^{\delta_{j}}, b_{j}^{\delta_{j}}\right)=(-\mathrm{i})(-1)^{\delta_{j}} \ell\left(x_{j} y_{j}\right) \mathrm{e}^{\mathrm{i} \theta_{j}} .
$$

When $\varepsilon$ is small we replace $x_{j} y_{j}$ by $d z_{j}^{\delta_{j}}$. Thus combining Eqs. (9) and (10) we obtain Eq. (8).

Lemma 14. When $\varepsilon$ is small and up to a term of order $\mathrm{O}(\varepsilon)$, expression (7) equals

$$
\frac{(-\mathrm{i})^{k}}{(2 \pi)^{k}} \sum_{\rho_{1}, \ldots, \rho_{k} \in\{0,1\}}\left[f_{w_{2}^{\delta_{2}} b_{1}^{\delta_{1}}}(0)\right]^{\rho_{1}} \cdots\left[f_{w_{1}^{\delta_{1}} b_{k}^{\delta_{k}}}(0)\right]^{\rho_{k}} F_{\rho_{1}}\left(b_{1}^{\delta_{1}}, w_{2}^{\delta_{2}}\right) \cdots F_{\rho_{k}}\left(b_{k}^{\delta_{k}}, w_{1}^{\delta_{1}}\right) \mathrm{d} z_{1}^{\delta^{1}} \ldots \mathrm{d} z_{k}^{\delta^{k}},
$$

where $F_{0}(z, w)=\frac{1}{z-w}, F_{1}(z, w)=F_{0}(\bar{z}, \bar{w})$, and the functions $f_{w b}$ are defined in Section 5.2. 
Proof. Let us drop the superscripts $\delta_{i}$. Plugging relation (8) in (7), we obtain

$$
(7)=(-\mathrm{i})^{k} \operatorname{sgn} \sigma K^{-1}\left(b_{1}, w_{2}\right) \cdots K^{-1}\left(b_{k}, w_{1}\right) \frac{1}{\varepsilon^{k}} \mathrm{~d} z_{1} \cdots \mathrm{d} z_{k}
$$

Moreover, for every $i \neq j, \lim _{\varepsilon \rightarrow 0} \frac{\left|b_{i}-w_{j}\right|}{\varepsilon}=\infty$, so that by Theorem 10 we have

$$
K^{-1}\left(b_{i}, w_{j}\right)=\frac{\varepsilon}{2 \pi}\left(F_{0}\left(b_{i}, w_{j}\right)+f_{w_{j} b_{i}}(0) F_{1}\left(b_{i}, w_{j}\right)\right)+\mathrm{O}\left(\varepsilon^{2}\right) .
$$

Eq. (11) is then (12) where the elements $K^{-1}\left(b_{i}, w_{j}\right)$ have been replaced by (13) and expanded out.

In what follows, all that we say is true whether the edge $w_{j}^{\delta_{j}} b_{j}^{\delta_{j}}$ has its black vertex on the right or on the left of the path $\gamma_{j}^{\varepsilon}$, that is whether $\delta_{j}=0$ or 1 . So to simplify notations, let us write $\left\{t_{j}\right\}$ instead of $\left\{\delta_{j} \in\{0,1\}, t_{j}^{\delta_{j}}\right\}$, hence $\left\{t_{j}\right\}$ is the set of indices which run along the path $\gamma_{j}^{\varepsilon}$. Keeping in mind that our aim is to take the limit as $\varepsilon \rightarrow 0$, we replace the vertices $b_{j}$ and $w_{j}$ in the argument of the function $F_{\rho_{j}}$ by one common vertex denoted by $z_{j}$. Define

$$
H\left(\rho_{1}, \ldots, \rho_{k}\right)=\sum_{t_{1}, \ldots, t_{k}}\left[f_{w_{2 t_{2}} b_{1 t_{1}}}(0)\right]^{\rho_{1}} \cdots\left[f_{w_{1 t_{1}} b_{k t_{k}}}(0)\right]^{\rho_{k}} F_{\rho_{1}}\left(z_{1 t_{1}}, z_{2 t_{2}}\right) \cdots F_{\rho_{k}}\left(z_{k t_{k}}, z_{1 t_{1}}\right) \mathrm{d} z_{1 t_{1}} \cdots \mathrm{d} z_{k t_{k}} .
$$

\section{Lemma 15.}

(1) If $\left(\rho_{1}, \ldots, \rho_{k}\right)=(0, \ldots, 0)$, then

$$
\lim _{\varepsilon \rightarrow 0} H(0, \ldots, 0)=\int_{\gamma_{1}} \ldots \int_{\gamma_{k}} F_{0}\left(z_{1}, z_{2}\right) \cdots F_{0}\left(z_{k}, z_{1}\right) \mathrm{d} z_{1} \cdots \mathrm{d} z_{k} .
$$

(2) If $\left(\rho_{1}, \ldots, \rho_{k}\right)=(1, \ldots, 1)$, then

$$
\lim _{\varepsilon \rightarrow 0} H(1, \ldots, 1)=(-1)^{k} \int_{\gamma_{1}} \ldots \int_{\gamma_{k}} F_{0}\left(\bar{z}_{1}, \bar{z}_{2}\right) \cdots F_{0}\left(\bar{z}_{k}, \bar{z}_{1}\right) \mathrm{d} \bar{z}_{1} \cdots \mathrm{d} \bar{z}_{k} .
$$

(3) Assume there exists $i \neq j \in\{1, \ldots, k\}$ such that $\rho_{i}=0, \rho_{j}=1$, then

$$
\lim _{\varepsilon \rightarrow 0}\left|H\left(\rho_{1}, \ldots, \rho_{k}\right)\right|=0 .
$$

Proof. Here are some preliminary notations. Dropping the second subscript, we consider an edge $x_{j} y_{j}$ of one of the paths $\gamma_{i}^{\varepsilon}$, where $x_{j}$ precedes $y_{j}$. Let us denote by $w_{j} b_{j}$ the dual edge of the edge $x_{j} y_{j}$, and let $\varepsilon \mathrm{e}^{\mathrm{i} \alpha_{j}}=y_{j}-w_{j}$, $\varepsilon \mathrm{e}^{\mathrm{i} \beta_{j}}=b_{j}-y_{j}$. With these notations, we have $d z_{j}=\varepsilon\left(\mathrm{e}^{\mathrm{i} \alpha_{j}}-\mathrm{e}^{\mathrm{i} \beta_{j}}\right)$ (see Fig. 4).

Moreover, define

$$
J\left(\rho_{1}, \ldots, \rho_{k}\right)=\left[f_{w_{2} b_{1}}(0)\right]^{\rho_{1}} \cdots\left[f_{w_{1} b_{k}}(0)\right]^{\rho_{k}} \mathrm{~d} z_{1} \cdots \mathrm{d} z_{k} .
$$

\section{Proof of (1).}

$$
J(0, \ldots, 0)=\mathrm{d} z_{1} \cdots \mathrm{d} z_{k},
$$

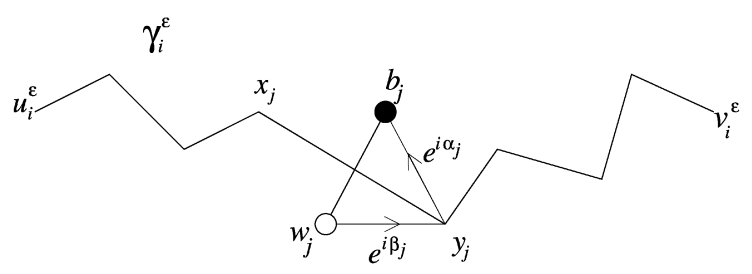

Fig. 4. Notations. 
so that

$$
H(0, \ldots, 0)=\sum_{t_{1}, \ldots, t_{k}} F_{0}\left(z_{1 t_{1}}, z_{2 t_{2}}\right) \cdots F_{0}\left(z_{k t_{k}}, z_{1 t_{1}}\right) \mathrm{d} z_{1 t_{1}} \cdots \mathrm{d} z_{k t_{k}} .
$$

Since the paths $\gamma_{j}$ are disjoint, the function $F_{0}\left(z_{1}, z_{2}\right) \cdots F_{0}\left(z_{k}, z_{1}\right)$ is integrable, and taking the limit as $\varepsilon \rightarrow 0$, we obtain (1).

\section{Proof of (2).}

$$
J(1, \ldots, 1)=f_{w_{2} b_{1}}(0) \cdots f_{w_{1} b_{k}}(0) \mathrm{d} z_{1} \cdots \mathrm{d} z_{k} .
$$

Fix a vertex $x$ of $G^{*}$. Then, by definition of the function $f_{w x}$,

$$
\begin{aligned}
J(1, \ldots, 1) & =f_{w_{2} x}(0) f_{x b_{1}}(0) \cdots f_{w_{1} x}(0) f_{x b_{k}}(0) \mathrm{d} z_{1} \cdots \mathrm{d} z_{k} \\
& =f_{w_{1} x}(0) f_{x b_{1}}(0) \cdots f_{w_{k} x}(0) f_{x b_{k}}(0) \mathrm{d} z_{1} \cdots \mathrm{d} z_{k} \\
& =f_{w_{1} b_{1}}(0) \cdots f_{w_{k} b_{k}}(0) \mathrm{d} z_{1} \cdots \mathrm{d} z_{k} .
\end{aligned}
$$

For every $j$, we have $f_{w_{j} b_{j}}(0)=\mathrm{e}^{-\mathrm{i}\left(\beta_{j}+\alpha_{j}\right)}$. Moreover, recall that $d z_{j}=\varepsilon\left(\mathrm{e}^{\mathrm{i} \alpha_{j}}-\mathrm{e}^{\mathrm{i} \beta_{j}}\right)$, so that $-\mathrm{d} \bar{z}_{j}=\mathrm{e}^{-\mathrm{i}\left(\beta_{j}+\alpha_{j}\right)} \mathrm{d} z_{j}$, and we deduce

$$
J(1, \ldots, 1)=(-1)^{k} \mathrm{~d} \bar{z}_{1} \cdots \mathrm{d} \bar{z}_{k} .
$$

This implies,

$$
H(1, \ldots, 1)=(-1)^{k} \sum_{t_{1}, \ldots, t_{k}} F_{0}\left(\bar{z}_{1 t_{1}}, \bar{z}_{2 t_{2}}\right) \cdots F_{0}\left(\bar{z}_{k t_{k}}, \bar{z}_{1 t_{1}}\right) \mathrm{d} \bar{z}_{1 t_{1}} \cdots \mathrm{d} \bar{z}_{k t_{k}} .
$$

Taking the limit as $\varepsilon \rightarrow 0$, we obtain (2).

Proof of (3). Consider $0<\ell<k$, and assume $\rho_{1}=\cdots=\rho_{\ell-1}=0, \rho_{\ell}=\cdots=\rho_{k}=1$. Let us prove that $\lim _{\varepsilon \rightarrow 0}|H(0, \ldots, 0,1, \ldots, 1)|=0$. Note that up to a permutation of indices, the argument is the same for the other cases.

$$
J(0, \ldots, 0,1, \ldots, 1)=f_{w_{\ell+1} b_{\ell}}(0) \cdots f_{w_{1} b_{k}}(0) \mathrm{d} z_{1} \cdots \mathrm{d} z_{k} .
$$

As above, let $x$ be a vertex of $G^{*}$. Then,

$$
J(0, \ldots, 0,1, \ldots, 1)=\left(f_{x b_{\ell}}(0) \mathrm{d} z \ell\right)\left(f_{w_{1} x}(0) \mathrm{d} z_{1}\right) \mathrm{d} z_{2} \cdots \mathrm{d} z_{\ell-1} \mathrm{~d} \bar{z}_{\ell+1} \cdots \mathrm{d} \bar{z}_{k} .
$$

Introducing the following notation

$$
\begin{aligned}
& H_{1}=F\left(z_{2 t_{2}}, z_{3 t_{3}}\right) \cdots F\left(z_{\ell-2 t_{\ell-2}}, z_{\ell-1 t_{\ell-1}}\right) F\left(\bar{z}_{\ell+1 t_{\ell+1}}, \bar{z}_{\ell+2 t_{\ell+2}}\right) \cdots F\left(\bar{z}_{k-1 t_{k-1}}, \bar{z}_{k t_{k}}\right), \\
& H_{2}=F\left(z_{1 t_{1}}, z_{2 t_{2}}\right) F\left(\bar{z}_{k t_{k}}, \bar{z}_{1 t_{1}}\right), \\
& H_{3}=F\left(z_{\ell-1 t_{\ell-1}}, z_{\ell t_{\ell}}\right) F\left(\bar{z}_{\ell t_{\ell}}, \bar{z}_{\ell+1 t_{\ell+1}}\right),
\end{aligned}
$$

we obtain

$$
\begin{aligned}
H & (0, \ldots, 0,1, \ldots, 1) \\
& =\sum_{t_{2}, \ldots, \hat{t}_{\ell}, \ldots, t_{k}} H_{1}\left(\sum_{t_{1}} H_{2} f_{w_{1 t_{1}} x}(0) \mathrm{d} z_{1 t_{1}}\right)\left(\sum_{t_{\ell}} H_{3} f_{x b_{\ell t_{\ell}}}(0) \mathrm{d} z_{\ell t_{\ell}}\right) \mathrm{d} z_{2 t_{2}} \cdots \mathrm{d} z_{\ell-1 t_{\ell-1}} \mathrm{~d} \bar{z}_{\ell+1 t_{\ell+1}} \cdots \mathrm{d} \bar{z}_{k t_{k}} .
\end{aligned}
$$

Let us prove

$$
\sum_{t_{\ell}} f_{x b_{\ell t_{\ell}}}(0) \mathrm{d} z z_{\ell t_{\ell}}=\mathrm{O}(\varepsilon)
$$

Dropping the second subscript, let $u_{\ell}^{\varepsilon}=x_{1}, y_{1}=x_{2}, y_{2}=x_{3}, \ldots, y_{m-1}=x_{m}, y_{m}=v_{\ell}^{\varepsilon}$ be the edge-path $\gamma_{\ell}^{\varepsilon}$. Denote by $\xi$ the quantity $f_{x x_{1}}(0)$, then

$$
f_{x b_{j}}(0)=\xi \mathrm{e}^{\mathrm{i}\left(\beta_{1}-\alpha_{1}\right)} \cdots \mathrm{e}^{\mathrm{i}\left(\beta_{j-1}-\alpha_{j-1}\right)} \mathrm{e}^{-\mathrm{i} \alpha_{j}} .
$$


Since $\mathrm{d} z_{j}=\varepsilon\left(\mathrm{e}^{\mathrm{i} \alpha_{j}}-\mathrm{e}^{\mathrm{i} \beta_{j}}\right)$, we obtain

$$
f_{x b_{j}}(0) \mathrm{d} z_{j}=\varepsilon \xi\left[\left(\mathrm{e}^{\mathrm{i}\left(\beta_{1}-\alpha_{1}\right)} \cdots \mathrm{e}^{\mathrm{i}\left(\beta_{j-1}-\alpha_{j-1}\right)}\right)-\left(\mathrm{e}^{\mathrm{i}\left(\beta_{1}-\alpha_{1}\right)} \cdots \mathrm{e}^{\mathrm{i}\left(\beta_{j-1}-\alpha_{j-1}\right)} \mathrm{e}^{\mathrm{i}\left(\beta_{j}-\alpha_{j}\right)}\right)\right],
$$

hence

$$
\begin{aligned}
\sum_{t_{\ell}} f_{x b_{\ell t_{\ell}}}(0) \mathrm{d} z_{\ell t_{\ell}} & =\sum_{j=1}^{m} f_{x b_{j}}(0) \mathrm{d} z_{j} \\
& =\varepsilon \xi\left[1-\mathrm{e}^{\mathrm{i}\left(\beta_{1}-\alpha_{1}\right)}+\sum_{j=2}^{m} \mathrm{e}^{\mathrm{i}\left(\beta_{1}-\alpha_{1}\right)} \cdots \mathrm{e}^{\mathrm{i}\left(\beta_{j-1}-\alpha_{j-1}\right)}-\left(\mathrm{e}^{\mathrm{i}\left(\beta_{1}-\alpha_{1}\right)} \cdots \mathrm{e}^{\mathrm{i}\left(\beta_{j-1}-\alpha_{j-1}\right)} \mathrm{e}^{\mathrm{i}\left(\beta_{j}-\alpha_{j}\right.}\right)\right] \\
& =\varepsilon \xi\left[1-\left(\mathrm{e}^{\mathrm{i}\left(\beta_{1}-\alpha_{1}\right)} \cdots \mathrm{e}^{\mathrm{i}\left(\beta_{m}-\alpha_{m}\right)}\right)\right] \quad \text { (telescopic sum). }
\end{aligned}
$$

We deduce $\left|\sum_{t_{\ell}} f_{x b_{\ell_{\ell}}}(0) \mathrm{d} z_{\ell_{\ell}}\right| \leqslant 2 \varepsilon$, and (14) is proved.

In a similar way we prove $\sum_{t_{1}} f_{w_{1 t_{1}} x}(0) \mathrm{d} z_{1 t_{1}}=\mathrm{O}(\varepsilon)$.

Using Taylor expansion in $\varepsilon$ for $H_{2}$ and $H_{3}$, we deduce that $\left(\sum_{t_{1}} H_{2} f_{w_{1 t_{1}} x}(0) \mathrm{d} z_{1 t_{1}}\right)$ and $\left(\sum_{t_{\ell}} H_{3} f_{x b_{\ell t_{\ell}}}(0) \mathrm{d} z \ell_{t_{\ell}}\right)$ are $\mathrm{O}(\varepsilon)$. Since the function $H_{1}$ is integrable, we conclude that $H(0, \ldots, 0,1, \ldots, 1)$ is $\mathrm{O}\left(\varepsilon^{2}\right)$ and so (3) is proved.

We now end the proof of Proposition 11. Rewriting the second subscript, and summing expression (7) over the paths $\gamma_{1}^{\varepsilon}, \ldots, \gamma_{k}^{\varepsilon}$, we obtain (by Lemmas 14 and 15):

$$
\begin{gathered}
\lim _{\varepsilon \rightarrow 0} \operatorname{sgn} \sigma \sum_{\delta_{1}, \ldots, \delta_{k} \in\{0,1\}} \sum_{t_{1}^{\delta_{1} \ldots t_{k}^{\delta_{k}}}}(-1)^{\delta_{1}+\cdots+\delta_{k}} a_{E} K^{-1}\left(b_{1 t_{1}^{\delta_{1}}}, w_{2 t_{2}^{\delta_{2}}}\right) \cdots K^{-1}\left(b_{k t_{k}^{\delta_{k}}}, w_{1 t_{1}^{\delta_{1}}}\right) \\
=\frac{(-\mathrm{i})^{k}}{(2 \pi)^{k}} \operatorname{sgn} \sigma \sum_{\rho=0,1}(-1)^{\rho k}\left(\int_{\gamma_{1}} \ldots \int_{\gamma_{k}} F_{0}\left(z_{1}^{\rho}, z_{2}^{\rho}\right) \cdots F_{0}\left(z_{k}^{\rho}, z_{1}^{\rho}\right) \mathrm{d} z_{1}^{\rho} \cdots \mathrm{d} z_{k}^{\rho}\right),
\end{gathered}
$$

where $z_{i}^{0}=z_{i}$ and $z_{i}^{1}=\bar{z}_{i}$. When $\sigma$ is a product of disjoint cycles, we can treat each cycle separately and the result is the product of terms like (15). Thus when we sum over all permutations with no fixed points, we obtain Eq. (3) of Proposition 11.

\section{Proposition 16.}

- When $k$ is odd, $\lim _{\varepsilon \rightarrow 0} \mathbb{E}\left[\left(h\left(v_{1}^{\varepsilon}\right)-h\left(u_{1}^{\varepsilon}\right)\right) \cdots\left(h\left(v_{k}^{\varepsilon}\right)-h\left(u_{k}^{\varepsilon}\right)\right)\right]=0$.

- When $k$ is even,

$$
\begin{aligned}
\lim _{\varepsilon \rightarrow 0} \mathbb{E}\left[\left(h\left(v_{1}^{\varepsilon}\right)-h\left(u_{1}^{\varepsilon}\right)\right) \cdots\left(h\left(v_{k}^{\varepsilon}\right)-h\left(u_{k}^{\varepsilon}\right)\right)\right] \\
\quad=\left(\frac{1}{\pi}\right)^{k / 2} \sum_{\tau \in \mathcal{T}_{k}} g\left(u_{\tau(1)}, v_{\tau(1)}, u_{\tau(2)}, v_{\tau(2)}\right) \cdots g\left(u_{\tau(k-1)}, v_{\tau(k-1)}, u_{\tau(k)}, v_{\tau(k)}\right),
\end{aligned}
$$

where $g\left(u, v, u^{\prime}, v^{\prime}\right)=g\left(v, v^{\prime}\right)+g\left(u, u^{\prime}\right)-g\left(v, u^{\prime}\right)-g\left(u, v^{\prime}\right), g$ is the Green function of the plane, and $\mathcal{T}_{k}$ is the set of all $(k-1) !$ ! pairings of $\{1, \ldots, k\}$.

Proof. Let us cite the following lemma from [11].

Lemma 17. [11] Let $M=\left(m_{i j}\right)$ be the $k \times k$ matrix defined by $m_{i i}=0$, and $m_{i j}=\frac{1}{\zeta_{i}-\zeta_{j}}$, when $i \neq j$. Then when $k$ is $o d d$, $\operatorname{det} M=0$, and when $k$ is even

$$
\operatorname{det} M=\sum_{\tau \in \mathcal{T}_{k}} \frac{1}{\left(\zeta_{\tau(1)}-\zeta_{\tau(2)}\right)^{2} \cdots\left(\zeta_{\tau(k-1)}-\zeta_{\tau(k)}\right)^{2}} .
$$


Combining Proposition 11 and Lemma 17, when $k=2$, we obtain

$$
\begin{aligned}
\lim _{\varepsilon \rightarrow 0} \mathbb{E}\left[\left(h\left(v_{1}^{\varepsilon}\right)-h\left(u_{1}^{\varepsilon}\right)\right)\left(h\left(v_{2}^{\varepsilon}\right)-h\left(u_{2}^{\varepsilon}\right)\right)\right]=-\frac{1}{4 \pi^{2}}\left(\int_{\gamma_{1}} \int_{\gamma_{2}} \frac{1}{\left(z_{1}-z_{2}\right)^{2}} \mathrm{~d} z_{1} \mathrm{~d} z_{2}+\int_{\gamma_{1}} \int_{\gamma_{2}} \frac{1}{\left(\bar{z}_{1}-\bar{z}_{2}\right)^{2}} \mathrm{~d} \bar{z}_{1} \mathrm{~d} \bar{z}_{2}\right) \\
=-\frac{1}{2 \pi^{2}} \log \left|\frac{\left(v_{1}-v_{2}\right)\left(u_{1}-u_{2}\right)}{\left(v_{1}-u_{2}\right)\left(u_{1}-v_{2}\right)}\right| \\
=\frac{1}{\pi} g\left(u_{1}, v_{1}, u_{2}, v_{2}\right) .
\end{aligned}
$$

The case of a general even $k$ is an easy but notationally cumbersome extension of the case $k=2$.

\subsection{Proof of Theorem 1}

\section{Proposition 18.}

$$
\lim _{\varepsilon \rightarrow 0} \mathbb{E}\left[\left(H^{\varepsilon} \varphi\right)^{k}\right]=\frac{1}{\pi^{k}} \mathbb{E}\left[(F \varphi)^{k}\right]= \begin{cases}0 & \text { when } k \text { is odd } \\ (k-1) ! ! \frac{1}{\pi^{k / 2}} G(\varphi, \varphi)^{k / 2} & \text { when } k \text { is even } .\end{cases}
$$

Proof. The second equality is just the $k$ th moment of a mean 0 , variance $\frac{1}{\sqrt{\pi}} G(\varphi, \varphi)$, Gaussian variable.

So let us prove equality between the first and the last term.

Consider $u_{1}, \ldots, u_{k}$ distinct points of $\mathbb{R}^{2}$, and for every $j$, let $u_{j}^{\varepsilon}$ be a vertex of $G^{\varepsilon}$ lying within $\mathrm{O}(\varepsilon)$ of $u_{j}$. Define

$$
H_{u_{j}}^{\varepsilon} \varphi=\sum_{v^{\varepsilon} \in G^{\varepsilon}} \varepsilon^{2} a\left(v^{*}\right) \varphi\left(v^{\varepsilon}\right)\left(h\left(v^{\varepsilon}\right)-h\left(u_{j}^{\varepsilon}\right)\right)=\sum_{v^{\varepsilon} \in K^{\varepsilon}} \varepsilon^{2} a\left(v^{*}\right) \varphi\left(v^{\varepsilon}\right)\left(h\left(v^{\varepsilon}\right)-h\left(u_{j}^{\varepsilon}\right)\right),
$$

where $K^{\varepsilon}=G^{\varepsilon} \cap K$, and $K=\operatorname{supp}(\varphi)$, then since we sum over a finite number of vertices,

$$
\begin{gathered}
\mathbb{E}\left[H_{u_{1}}^{\varepsilon} \varphi \cdots H_{u_{k}}^{\varepsilon} \varphi\right]=\mathbb{E}\left[\sum_{v_{1}^{\varepsilon} \in K^{\varepsilon}} \varepsilon^{2} a\left(v_{1}^{*}\right) \varphi\left(v_{1}^{\varepsilon}\right)\left(h\left(v_{1}^{\varepsilon}\right)-h\left(u_{1}^{\varepsilon}\right)\right) \cdots \sum_{v_{k}^{\varepsilon} \in K^{\varepsilon}} \varepsilon^{2} a\left(v_{k}^{*}\right) \varphi\left(v_{k}^{\varepsilon}\right)\left(h\left(v_{k}^{\varepsilon}\right)-h\left(u_{k}^{\varepsilon}\right)\right)\right] \\
=\sum_{v_{1}^{\varepsilon} \in K^{\varepsilon}} \cdots \sum_{v_{k}^{\varepsilon} \in K^{\varepsilon}}\left(\varepsilon^{2}\right)^{k} a\left(v_{1}^{*}\right) \cdots a\left(v_{k}^{*}\right) \varphi\left(v_{1}^{\varepsilon}\right) \cdots \varphi\left(v_{k}^{\varepsilon}\right) \mathbb{E}\left[\left(h\left(v_{1}^{\varepsilon}\right)-h\left(u_{1}^{\varepsilon}\right)\right) \cdots\left(h\left(v_{k}^{\varepsilon}\right)-h\left(u_{k}^{\varepsilon}\right)\right)\right] .
\end{gathered}
$$

Lemma 19. As $\varepsilon \rightarrow 0$, the Riemann sum (17) converges to

$$
\int_{\mathbb{R}^{2}} \ldots \int_{\mathbb{R}^{2}} \varphi\left(v_{1}\right) \ldots \varphi\left(v_{k}\right) \lim _{\varepsilon \rightarrow 0} \mathbb{E}\left[\left(h\left(v_{1}^{\varepsilon}\right)-h\left(u_{1}^{\varepsilon}\right)\right) \cdots\left(h\left(v_{k}^{\varepsilon}\right)-h\left(u_{k}^{\varepsilon}\right)\right)\right] \mathrm{d} v_{1} \cdots \mathrm{d} v_{k},
$$

where $\lim _{\varepsilon \rightarrow 0} \mathbb{E}\left[\left(h\left(v_{1}^{\varepsilon}\right)-h\left(u_{1}^{\varepsilon}\right)\right) \cdots\left(h\left(v_{k}^{\varepsilon}\right)-h\left(u_{k}^{\varepsilon}\right)\right)\right]$ is given by Proposition 16.

Proof. In what follows, all that we say is true whether $\delta_{j}=0$ or 1 , so to simplify notations, as before, let us write $\left\{t_{j}\right\}$ instead of $\left\{\delta_{j} \in\{0,1\}, t_{j}^{\delta_{j}}\right\}$, hence $\left\{t_{j}\right\}$ is the set of indices which run along the path $\gamma_{j}^{\varepsilon}$. Combining Eqs. (4), (6) and (8) yields,

$$
\begin{aligned}
\mathbb{E} & {\left[h\left(v_{1}^{\varepsilon}\right)-h\left(u_{1}^{\varepsilon}\right)\right] \cdots\left[h\left(v_{k}^{\varepsilon}\right)-h\left(u_{k}^{\varepsilon}\right)\right] } \\
& =(-\mathrm{i})^{k} \sum_{t_{1}, \ldots, t_{k}} \sum_{\sigma \in \widetilde{S}_{k}} \operatorname{sgn} \sigma K^{-1}\left(b_{1 t_{1}}, w_{\sigma(1) t_{\sigma(1)}}\right) \cdots K^{-1}\left(b_{k t_{k}}, w_{\sigma(k) t_{\sigma(k)}}\right) \frac{1}{\varepsilon^{k}} \mathrm{~d} z_{1 t_{1}} \cdots \mathrm{d} z_{k t_{k}} .
\end{aligned}
$$

It suffices to consider the case where $\sigma$ is a $k$-cycle, other cases are treated similarly. Indices are denoted cyclically (i.e. $k+1 \equiv 1$ ). There is a singularity in (18) as soon as $v_{j}^{\varepsilon}=v_{j+1}^{\varepsilon}$ for some indices $j$. Hence, we need to prove that for $\varepsilon$ small enough,

$$
\sum_{v_{1}^{\varepsilon}} \cdots \sum_{v_{k}^{\varepsilon}}\left(\varepsilon^{2}\right)^{k} a\left(v_{1}^{\varepsilon}\right) \cdots a\left(v_{k}^{\varepsilon}\right)\left|\varphi\left(v_{1}^{\varepsilon}\right) \cdots \varphi\left(v_{k}^{\varepsilon}\right)\right| \sum_{t_{1}, \ldots, t_{k}}\left|K^{-1}\left(b_{1 t_{1}}, w_{2 t_{2}}\right) \cdots K^{-1}\left(b_{k t_{k}}, w_{1 t_{1}}\right)\right| \frac{1}{\varepsilon^{k}} \mathrm{~d} z_{1 t_{1}} \cdots \mathrm{d} z_{k t_{k}},
$$


is $\mathrm{o}(1)$, when the sum is over vertices $v_{1}^{\varepsilon}, \ldots, v_{k}^{\varepsilon}$ that satisfy

$$
\left|v_{1}^{\varepsilon}-v_{2}^{\varepsilon}\right| \leqslant \delta, \ldots,\left|v_{m}^{\varepsilon}-v_{m+1}^{\varepsilon}\right| \leqslant \delta,\left|v_{m+1}^{\varepsilon}-v_{m+2}^{\varepsilon}\right|>\delta, \ldots,\left|v_{k}^{\varepsilon}-v_{1}^{\varepsilon}\right|>\delta,
$$

for some $1 \leqslant m<k-1$. Let $0<\beta<1$, up to a renaming of indices, this amounts to considering vertices $v_{1}^{\varepsilon}, \ldots, v_{k}^{\varepsilon}$ in $\Theta_{1} \cap \Theta_{2} \cap \Theta_{3}$, where

$$
\begin{aligned}
& \Theta_{1}=\left\{v_{1}^{\varepsilon}, \ldots, v_{m}^{\varepsilon}|1 \leqslant i \leqslant m, 0 \leqslant| v_{i}^{\varepsilon}-v_{i+1}^{\varepsilon} \mid \leqslant \varepsilon^{\beta}\right\}, \\
& \Theta_{2}=\left\{v_{m+1}^{\varepsilon}, \ldots, v_{n}^{\varepsilon}\left|m+1 \leqslant j \leqslant n, \varepsilon^{\beta} \leqslant\right| v_{j}^{\varepsilon}-v_{j+1}^{\varepsilon} \mid \leqslant \delta\right\}, \\
& \Theta_{3}=\left\{v_{n+1}^{\varepsilon}, \ldots, v_{k}^{\varepsilon}|n+1 \leqslant \ell \leqslant k,| v_{\ell}^{\varepsilon}-v_{\ell+1}^{\varepsilon} \mid>\delta\right\},
\end{aligned}
$$

for some $1 \leqslant m<n<k-1$.

Since $u_{1}, \ldots, u_{k}$ are distinct vertices of $\mathbb{R}^{2}$, and since Eq. (19) does not depend on the path $\gamma_{j}^{\varepsilon}$ from $u_{j}^{\varepsilon}$ to $v_{j}^{\varepsilon}$, let us choose the paths $\gamma_{1}^{\varepsilon}, \ldots, \gamma_{k}^{\varepsilon}$ as follows. Note that it suffices to consider the part of the path $\gamma_{j}^{\varepsilon}$ where the vertices $b_{j t_{j}}$ and $w_{j t_{j}}$ are within distance $\delta$ from $v_{j}^{\varepsilon}$. Let us write $\left|t_{j}\right| \leqslant \delta$ to denote indices $t_{j}$ which refer to vertices of $\gamma_{j}^{\varepsilon}$ that are at distance at most $\delta$ from $v_{j}^{\varepsilon}$. Take $\gamma_{j}^{\varepsilon}$ to approximate a straight line within $\mathrm{O}(\varepsilon)$, from $v_{j}^{\varepsilon}$ to $v_{j}^{\varepsilon}+\delta$. Moreover, ask that if one continues the lines of $\gamma_{j}^{\varepsilon}$ and $\gamma_{j+1}^{\varepsilon}$ away from $u_{j}^{\varepsilon}$ and $u_{j+1}^{\varepsilon}$, they intersect and form an angle $\theta_{j}$. Let us use the definition of the paths $\gamma_{j}^{\varepsilon}$, and consider the three following cases. Whenever it is not confusing, we shall drop the second subscript. $C$ denotes a generic constant, $A \sim B$ means $A$ and $B$ are of the same order.

- $v_{1}^{\varepsilon}, \ldots, v_{m}^{\varepsilon} \in \Theta_{1}$.

By Remark 20 below, we have $\left|K^{-1}\left(b_{i}, w_{l+1}\right)\right| \leqslant C$. This implies,

$$
\sum_{\left|t_{1}\right| \leqslant \delta, \ldots,\left|t_{m}\right| \leqslant \delta}\left|K^{-1}\left(b_{1 t_{1}}, w_{2 t_{2}}\right) \cdots K^{-1}\left(b_{m t_{m}}, w_{m+1 t_{m+1}}\right)\right| \frac{1}{\varepsilon^{m}} \mathrm{~d} z_{1 t_{1}} \cdots \mathrm{d} z_{m t_{m}} \leqslant\left(\frac{C \delta}{\varepsilon}\right)^{m} .
$$

- $v_{n+1}^{\varepsilon}, \ldots, v_{k}^{\varepsilon} \in \Theta_{3}$.

By definition of the paths $\gamma_{\ell}^{\varepsilon},\left|b_{\ell}-w_{\ell+1}\right|>\delta$, so that $\lim _{\varepsilon \rightarrow 0} \frac{\left|b_{\ell}-w_{\ell+1}\right|}{\varepsilon}=\infty$. Using Theorem 10 yields

$$
\frac{1}{\varepsilon}\left|K^{-1}\left(b_{\ell}, w_{\ell+1}\right)\right|=\mathrm{O}\left(\frac{1}{\left|b_{\ell}-w_{\ell+1}\right|}\right) \leqslant \frac{C}{\delta} .
$$

Hence,

$$
\sum_{\left|t_{n+1}\right| \leqslant \delta, \ldots,\left|t_{k}\right| \leqslant \delta}\left|K^{-1}\left(b_{n+1 t_{n+1}}, w_{n+2 t_{n+2}}\right) \cdots K^{-1}\left(b_{k t_{k}}, w_{1 t_{1}}\right)\right| \frac{1}{\varepsilon^{k-n}} \mathrm{~d} z_{n+1 t_{n+1}} \cdots \mathrm{d} z_{k t_{k}} \leqslant\left(\frac{C}{\delta}\right)^{k-n} .
$$

- $v_{m+1}^{\varepsilon}, \ldots, v_{n}^{\varepsilon} \in \Theta_{2}$.

Let $\varepsilon^{\beta} \leqslant L \leqslant \delta$, and define the annulus, $A\left(v_{j}^{\varepsilon}, L\right)=\left\{v \in G^{\varepsilon}|L \leqslant| v-v_{j}^{\varepsilon} \mid \leqslant L+\varepsilon\right\}$. By definition of the paths $\gamma_{j}^{\varepsilon}$, if $v_{j}^{\varepsilon} \in A\left(v_{j+1}^{\varepsilon}, L\right)$, we have $\lim _{\varepsilon \rightarrow 0} \frac{\left|b_{j}-w_{j+1}\right|}{\varepsilon}=\infty$ (since $\beta<1$ ). Using Theorem 10 yields

$$
\frac{1}{\varepsilon}\left|K^{-1}\left(b_{j}, w_{j+1}\right)\right|=\mathrm{O}\left(\frac{1}{\left|b_{j}-w_{j+1}\right|}\right) \leqslant \frac{1}{\min _{\left\{w_{j+1} \in \gamma_{j+1}^{\varepsilon}\right\}}\left|b_{j}-w_{j+1}\right|}=\frac{C}{x_{j}+C L} \sin \theta_{j},
$$

where $x_{j}$ is the distance from $v_{j}^{\varepsilon}$ to $b_{j}$. Let us replace $\sin \theta_{j}$ by $C$. Hence, if for $m+1 \leqslant j \leqslant n, v_{j}^{\varepsilon} \in A\left(v_{j+1}, L_{j}\right)$, we obtain

$$
\begin{aligned}
& \quad \sum_{\left|t_{m+1}\right| \leqslant \delta, \ldots,\left|t_{n}\right| \leqslant \delta}\left|K^{-1}\left(b_{m+1 t_{m+1}}, w_{m+2 t_{m+2}}\right) \cdots K^{-1}\left(b_{n t_{n}}, w_{n+1 t_{n+1}}\right)\right| \frac{1}{\varepsilon^{n-m}} \mathrm{~d} z_{m+1 t_{m+1}} \cdots \mathrm{d} z_{n t_{n}} \\
& \leqslant \sum_{\left|t_{m+1}\right| \leqslant \delta, \ldots,\left|t_{n}\right| \leqslant \delta} \frac{C}{x_{m+1 t_{m+1}}+C L_{m+1}} \cdots \frac{C}{x_{n t_{n}}+C L_{n}} \mathrm{~d} x_{m+1 t_{m+1}} \cdots \mathrm{d} x_{n t_{n}} \\
& \sim C \prod_{j=m+1}^{n} \log \left(\frac{\delta+C L_{j}}{C L_{j}}\right) .
\end{aligned}
$$


Let $\Xi$ be the sum (19) over vertices $v_{1}^{\varepsilon}, \ldots, v_{k}^{\varepsilon} \in \Theta_{1} \cap \Theta_{2} \cap \Theta_{3}$. Denote by $M=\sup _{v \in \mathbb{R}^{2}}|\varphi(v)|$. Then, $\Xi \leqslant \Xi_{1} \Xi_{2} \Xi_{3}$, where

$$
\begin{aligned}
& \Xi_{1}=C M^{m}\left[\sum_{v_{1}^{\varepsilon}, \ldots, v_{m}^{\varepsilon} \in \Theta_{1}} \varepsilon^{2 m}\left(\frac{\delta}{\varepsilon}\right)^{m}\right], \\
& \Xi_{2}=\left[\sum_{v_{n+1}^{\varepsilon}, \ldots, v_{k}^{\varepsilon} \in \Theta_{2}} \varepsilon^{2(k-n)}\left|\varphi\left(v_{n+1}^{\varepsilon}\right)\right| \cdots\left|\varphi\left(v_{k}^{\varepsilon}\right)\right|\left(\frac{1}{\delta}\right)^{k-n}\right], \\
& \Xi_{3}=C M^{n-m}\left[\sum_{L_{m+1}=0}^{\delta} \cdots \sum_{L_{n}=0}^{\delta} \varepsilon^{n-m} L_{m+1} \cdots L_{n} \log \left(\frac{\delta+C L_{m+1}}{L_{m+1}}\right) \cdots \log \left(\frac{\delta+C L_{n}}{L_{n}}\right) \mathrm{d} L_{m+1} \cdots \mathrm{d} L_{n}\right] .
\end{aligned}
$$

Moreover,

$$
\begin{aligned}
& \Xi_{1} \leqslant C M^{m} \delta^{m} \varepsilon^{m(2 \beta-1)}, \\
& \Xi_{2} \leqslant\left(\frac{1}{\delta}\right)^{k-n} \sum_{v_{n+1}^{\varepsilon}, \ldots, v_{k}^{\varepsilon} \in \mathbb{R}^{2}} \varepsilon^{2(k-n)}\left|\varphi\left(v_{n+1}^{\varepsilon}\right)\right| \cdots\left|\varphi\left(v_{k}^{\varepsilon}\right)\right| \leqslant M^{k-n}\left(\frac{1}{\delta}\right)^{k-n}, \\
& \Xi_{3} \sim C M^{n-m}(\delta \log \delta)^{n-m} .
\end{aligned}
$$

Hence, $\Xi \leqslant C M^{k} \delta^{2 n-k} \varepsilon^{m(2 \beta-1)}$. Let us take $\beta=2 / 3<1$, then $m(2 \beta-1)>0$. If $2 n-k \geqslant 1$, then $\Xi=o(1)$. If $2 n-k \leqslant 0$, take $\varepsilon \leqslant \delta^{\frac{k-2 n+1}{m(2 \beta-1)}}$, and $\Xi=\mathrm{o}(1)$.

Remark 20. Let $G^{*}$ be a bipartite isoradial graph, and let $K^{-1}$ be the corresponding inverse Dirac operator, then for every black vertex $b$ and every white vertex $w$ of $G^{*}$, we have

$$
\left|K^{-1}(b, w)\right| \leqslant \mathcal{C}
$$

for some constant $\mathcal{C}$ which only depends on the graph $G^{*}$.

Proof. By Theorem 4.2 of [13], $K^{-1}$ is given by

$$
K^{-1}(b, w)=\frac{1}{4 \pi^{2} \mathrm{i}} \int_{C} f_{w b}(z) \log z \mathrm{~d} z
$$

where $C$ is a closed contour surrounding counterclockwise the part of the circle $\left\{\mathrm{e}^{\mathrm{i} \theta} \mid \theta \in\left[\theta_{0}-\pi+\Delta, \theta_{0}+\pi-\Delta\right]\right\}$, which contains all the poles of $f_{w b}$, and with the origin in its exterior. Without loss of generality suppose $\theta_{0}=0$. As in [13], let us homotope the curve $C$ to the curve from $-\infty$ to the origin and back to $-\infty$ along the two sides of the negative real axis. On the two sides of this ray, $\log z$ differs by $2 \pi \mathrm{i}$, hence

$$
K^{-1}(b, w)=\frac{1}{2 \pi} \int_{-\infty}^{0} f_{w b}(t) \mathrm{d} t, \quad \text { where } f_{w b}(t)=\frac{1}{\left(t-\mathrm{e}^{\mathrm{i} \theta_{1}}\right)\left(t-\mathrm{e}^{\mathrm{i} \theta_{2}}\right)} \prod_{j=1}^{k} \frac{\left(t-\mathrm{e}^{\mathrm{i} \alpha_{j}}\right)}{\left(t-\mathrm{e}^{\mathrm{i} \beta_{j}}\right)} .
$$

Refer to [13] for the choice of path from $w$ to $b$, that is for the definition of the angles $\theta_{1}, \theta_{2}, \alpha_{j}, \beta_{j}$. These angles have the property that for all $j, \cos \alpha_{j} \leqslant \cos \beta_{j}$, so

$$
\left|\frac{t-\mathrm{e}^{\mathrm{i} \theta_{j}}}{t-\mathrm{e}^{\mathrm{i} \beta_{j}}}\right| \leqslant 1
$$

For every $\mathrm{e}^{\mathrm{i} \theta} \in\left\{\mathrm{e}^{\mathrm{i} \theta} \mid \theta \in\left[\theta_{0}-\pi+\Delta, \theta_{0}+\pi-\Delta\right]\right\}$, and for every $t<0$, we have

$$
\left|t-\mathrm{e}^{\mathrm{i} \theta}\right|^{2} \geqslant\left|t-\mathrm{e}^{\mathrm{i}(\pi+\Delta)}\right|^{2}=\left|t+\mathrm{e}^{\mathrm{i} \Delta}\right|^{2} .
$$


Moreover for every $t \in \mathbb{R}$, we have $\left|t+\mathrm{e}^{\mathrm{i} \Delta}\right|^{2} \geqslant \sin ^{2} \Delta$, and $\left|t+\mathrm{e}^{\mathrm{i} \Delta}\right|^{2} \geqslant(t+1)^{2}$, thus

$$
\int_{-\infty}^{0}\left|f_{w b}(t)\right| \mathrm{d} t \leqslant \int_{-\infty}^{-2} \frac{1}{(t+1)^{2}} \mathrm{~d} t+\int_{-2}^{0} \frac{1}{\sin ^{2} \Delta} \mathrm{d} t=1+\frac{2}{\sin ^{2} \Delta} .
$$

Hence $\left|K^{-1}(b, w)\right| \leqslant \mathcal{C}$, where $\mathcal{C}=\frac{1}{2 \pi}\left(1+\frac{2}{\sin ^{2} \Delta}\right)$.

We end the proof of Proposition 18 with the following

\section{Lemma 21.}

(1) $\int_{\mathbb{R}^{2}} \cdots \int_{\mathbb{R}^{2}} \varphi\left(v_{1}\right) \cdots \varphi\left(v_{k}\right) \lim _{\varepsilon \rightarrow 0} \mathbb{E}\left[\left(h\left(v_{1}^{\varepsilon}\right)-h\left(u_{1}^{\varepsilon}\right)\right) \cdots\left(h\left(v_{k}^{\varepsilon}\right)-h\left(u_{k}^{\varepsilon}\right)\right)\right] \mathrm{d} z_{1} \cdots \mathrm{d} z_{k}$

$$
= \begin{cases}0 & \text { when } k \text { is odd, } \\ (k-1) ! ! \frac{1}{\pi^{k / 2}} G(\varphi, \varphi)^{k / 2} & \text { when } k \text { is even. }\end{cases}
$$

(2) $\lim _{\varepsilon \rightarrow 0} \mathbb{E}\left[H_{u_{1}}^{\varepsilon} \varphi \cdots H_{u_{k}}^{\varepsilon} \varphi\right]=\lim _{\varepsilon \rightarrow 0} \mathbb{E}\left[\left(H^{\varepsilon} \varphi\right)^{k}\right]$.

Proof. (1) is deduced from the formula of Proposition 16, and from the fact that $\varphi$ is a mean 0 function.

(2) is a consequence of the fact that $\varphi$ is a mean 0 function, and of estimates of the kind of those of Lemma 19.

The proof of Proposition 18 is complete.

\subsection{Remark}

Note that the double periodicity assumption of the graph $G^{*}$ is only required in Lemma 12, where we implicitly use the expression of Theorem 3 for the Gibbs measure $\mu$, as a function of the Dirac operator $K$, and its inverse $K^{-1}$. By Remark 4, this assumption can be released for the measure $\mu$, in the case where the graph $G$ is a lozenge-withdiagonals tiling. Hence, Theorem 1 remains valid when the graph $G$ is any lozenge-with-diagonals tiling of the plane, periodic or not.

\section{Proof of Corollary 2}

We place ourselves in the context of Corollary 2: $\mathcal{Q}$ is the set of triangular quadri-tilings, and assume quadri-tiles are assigned the critical weight function. Recall the following notations: $\mathbb{T}^{\varepsilon}$ is the equilateral triangular lattice whose edge-lengths have been multiplied by $\varepsilon, \mathbb{P}$ is the Gibbs measure on $\mathcal{Q}$ of Section 2.4; for $i=1,2$,

$$
H_{i}^{\varepsilon} \varphi=\varepsilon^{2} \sum_{v \in V\left(\mathbb{T}^{\varepsilon}\right)} \frac{\sqrt{3}}{2} \varphi(v) h_{i}^{\varepsilon}(v),
$$

and $F_{1}, F_{2}$ are Gaussian free fields in the plane.

In order to prove weak convergence in distribution of the height functions $h_{1}^{\varepsilon}$ and $h_{2}^{\varepsilon}$ to two independent Gaussian free fields $F_{1}$ and $F_{2}$, it suffices to show that, $\forall \varphi \in C_{c, 0}^{\infty}\left(\mathbb{R}^{2}\right)$ :

$$
\lim _{\varepsilon \rightarrow 0} \mathbb{E}\left[\left(H_{1}^{\varepsilon} \varphi\right)^{k}\left(H_{2}^{\varepsilon} \varphi\right)^{m}\right]=\mathbb{E}\left[\left(F_{1} \varphi\right)^{k}\right] \mathbb{E}\left[\left(F_{2} \varphi\right)^{m}\right] .
$$

The key point is to obtain the analog of the moment formula of Proposition 11. The rest of the proof goes through in the same way, and since notations are quite heavy, we do not repeat it here.

The idea to obtain the moment formula is the following. Recall that triangular quadri-tilings correspond to two superimposed dimer models, the first on lozenge-with-diagonals tilings of $\mathcal{L}$ and the second on the equilateral triangular lattice $\mathbb{T}$. Recall also that both lozenge-with-diagonals tilings and $\mathbb{T}$ are isoradial graphs. Hence, we start by applying Proposition 11 in the case where the graph $G$ is a lozenge-with-diagonals tiling $L \in \mathcal{L}$. Then in Lemma 23, 
we prove some uniformity of convergence for every $L \in \mathcal{L}$, and we conclude the proof by using Proposition 11 in the case where the graph $G$ is the equilateral triangular lattice $\mathbb{T}$.

Let $u_{1}, \ldots, u_{k}, v_{1}, \ldots, v_{k}, \mathrm{u}_{1}, \ldots, \mathrm{u}_{m}, \mathrm{v}_{1}, \ldots, \mathrm{v}_{m}$ be distinct points of $\mathbb{R}^{2}$, and let $\gamma_{1}, \ldots, \gamma_{k}, \gamma_{1}^{\prime}, \ldots, \gamma_{m}^{\prime}$ be pairwise disjoint, simple, smooth paths such that $\gamma_{j}$ (resp. $\gamma_{j}^{\prime}$ ) runs from $u_{j}$ to $v_{j}$ (resp. from $\mathrm{u}_{j}$ to $v_{j}$ ). Define

$$
\mathcal{G}\left(u_{1}, v_{1}, \ldots, u_{k}, v_{k}\right)=\frac{(-\mathrm{i})^{k}}{(2 \pi)^{k}} \sum_{\varepsilon=0,1}(-1)^{k \varepsilon}\left(\int_{\gamma_{1}} \ldots \int_{\substack { \gamma_{k} \\
\begin{subarray}{c}{i, j \in[1, k] \\
i \neq j{ \gamma _ { k } \\
\begin{subarray} { c } { i , j \in [ 1 , k ] \\
i \neq j } }\end{subarray}}\left(\frac{1}{z_{i}^{\varepsilon}-z_{j}^{\varepsilon}}\right) \mathrm{d} z_{1}^{\varepsilon} \cdots \mathrm{d} z_{k}^{\varepsilon}\right) .
$$

Similarly, define $\mathcal{G}\left(\mathrm{u}_{1}, \mathrm{v}_{1}, \ldots, \mathrm{u}_{m}, \mathrm{v}_{m}\right)$. Let $u_{j}^{\varepsilon}, v_{j}^{\varepsilon}, \mathrm{u}_{j}^{\varepsilon}, \mathrm{v}_{j}^{\varepsilon}$ be vertices of $\mathbb{T}^{\varepsilon}$ lying within $\mathrm{O}(\varepsilon)$ of $u_{j}, v_{j}, \mathrm{u}_{j}, \mathrm{v}_{j}$ respectively.

\section{Lemma 22.}

$$
\begin{aligned}
& \lim _{\varepsilon \rightarrow 0} \mathbb{E}\left[\left(h_{1}\left(v_{1}^{\varepsilon}\right)-h_{1}\left(u_{1}^{\varepsilon}\right)\right) \cdots\left(h_{1}\left(v_{k}^{\varepsilon}\right)-h_{1}\left(u_{k}^{\varepsilon}\right)\right)\left(h_{2}\left(\mathrm{v}_{1}^{\varepsilon}\right)-h_{2}\left(\mathrm{u}_{1}^{\varepsilon}\right)\right) \cdots\left(h_{2}\left(\mathrm{v}_{m}^{\varepsilon}\right)-h_{2}\left(\mathrm{u}_{m}^{\varepsilon}\right)\right)\right] \\
& \quad=\mathcal{G}\left(u_{1}, v_{1}, \ldots, u_{k}, v_{k}\right) \mathcal{G}\left(\mathrm{u}_{1}, \mathrm{v}_{1}, \ldots, \mathrm{u}_{m}, \mathrm{v}_{m}\right) .
\end{aligned}
$$

Proof. By definition of the measure $\mathbb{P}$, we have:

$$
\begin{aligned}
\mathbb{E} & {\left[\left(h_{1}\left(v_{1}^{\varepsilon}\right)-h_{1}\left(u_{1}^{\varepsilon}\right)\right) \cdots\left(h_{1}\left(v_{k}^{\varepsilon}\right)-h_{1}\left(u_{k}^{\varepsilon}\right)\right)\left(h_{2}\left(\mathrm{v}_{1}^{\varepsilon}\right)-h_{2}\left(\mathrm{u}_{1}^{\varepsilon}\right)\right) \cdots\left(h_{2}\left(\mathrm{v}_{m}^{\varepsilon}\right)-h_{2}\left(\mathrm{u}_{m}^{\varepsilon}\right)\right)\right] } \\
& =\sum_{\mathrm{L}^{*} \in \mathcal{M}\left(\mathbb{T}^{*}\right)} \mathbb{E}_{\mu^{L}}\left[\left(h_{1}\left(v_{1}^{\varepsilon}\right)-h_{1}\left(u_{1}^{\varepsilon}\right)\right) \cdots\left(h_{1}\left(v_{k}^{\varepsilon}\right)-h_{1}\left(u_{k}^{\varepsilon}\right)\right)\left(h_{2}\left(\mathrm{v}_{1}^{\varepsilon}\right)-h_{2}\left(\mathrm{u}_{1}^{\varepsilon}\right)\right) \cdots\left(h_{2}\left(\mathrm{v}_{m}^{\varepsilon}\right)-h_{2}\left(\mathrm{u}_{m}^{\varepsilon}\right)\right)\right] \mathrm{d} \mu^{\mathbb{T}}\left(\mathrm{L}^{*}\right) \\
& =\sum_{\mathrm{L}^{*} \in \mathcal{M}\left(\mathbb{T}^{*}\right)}\left(h_{2}\left(\mathrm{v}_{1}^{\varepsilon}\right)-h_{2}\left(\mathrm{u}_{1}^{\varepsilon}\right)\right) \cdots\left(h_{2}\left(\mathrm{v}_{m}^{\varepsilon}\right)-h_{2}\left(\mathrm{u}_{m}^{\varepsilon}\right)\right) \mathbb{E}_{\mu^{L}}\left[\left(h_{1}\left(v_{1}^{\varepsilon}\right)-h_{1}\left(u_{1}^{\varepsilon}\right)\right) \cdots\left(h_{1}\left(v_{k}^{\varepsilon}\right)-h_{1}\left(u_{k}^{\varepsilon}\right)\right)\right] \mathrm{d} \mu^{\mathbb{T}}\left(\mathrm{L}^{*}\right) .
\end{aligned}
$$

Using Section 5.5, we can use Proposition 11 in the case where $G$ is any lozenge-with-diagonals tiling $L \in \mathcal{L}$ (periodic or not), hence for every $L \in \mathcal{L}$, we have:

$$
\lim _{\varepsilon \rightarrow 0} \mathbb{E}_{\mu^{L}}\left[\left(h_{1}\left(v_{1}^{\varepsilon}\right)-h_{1}\left(u_{1}^{\varepsilon}\right)\right) \cdots\left(h_{1}\left(v_{k}^{\varepsilon}\right)-h_{1}\left(u_{k}^{\varepsilon}\right)\right)\right]=\mathcal{G}\left(u_{1}, v_{1}, \ldots, u_{k}, v_{k}\right) .
$$

Note that the right-hand side is independent of $L$, hence to obtain Lemma 22, we need to prove that convergence in (20) is uniform in $L$, see Lemma 23 below. Indeed, assuming this is the case, for $\varepsilon$ small, we can write:

$$
\begin{aligned}
& \sum_{\mathrm{L}^{*} \in \mathcal{M}\left(\mathbb{T}^{*}\right)}\left(h_{2}\left(\mathrm{v}_{1}^{\varepsilon}\right)-h_{2}\left(\mathrm{u}_{1}^{\varepsilon}\right)\right) \cdots\left(h_{2}\left(\mathrm{v}_{m}^{\varepsilon}\right)-h_{2}\left(\mathrm{u}_{m}^{\varepsilon}\right)\right) \mathbb{E}_{\mu^{L}}\left[\left(h_{1}\left(v_{1}^{\varepsilon}\right)-h_{1}\left(u_{1}^{\varepsilon}\right)\right) \cdots\left(h_{1}\left(v_{k}^{\varepsilon}\right)-h_{1}\left(u_{k}^{\varepsilon}\right)\right)\right] \mathrm{d} \mu^{\mathbb{T}}\left(\mathrm{L}^{*}\right) \\
= & \sum_{\mathrm{L}^{*} \in \mathcal{M}\left(\mathbb{T}^{*}\right)}\left(h_{2}\left(\mathrm{v}_{1}^{\varepsilon}\right)-h_{2}\left(\mathrm{u}_{1}^{\varepsilon}\right)\right) \cdots\left(h_{2}\left(\mathrm{v}_{m}^{\varepsilon}\right)-h_{2}\left(\mathrm{u}_{m}^{\varepsilon}\right)\right)\left(\mathcal{G}\left(u_{1}, v_{1}, \ldots, u_{k}, v_{k}\right)+\mathrm{O}(\varepsilon)\right) \mathrm{d} \mu^{\mathbb{T}}\left(\mathrm{L}^{*}\right) \\
= & \left(\mathcal{G}\left(u_{1}, v_{1}, \ldots, u_{k}, v_{k}\right)+\mathrm{O}(\varepsilon)\right) \sum_{\mathrm{L}^{*} \in \mathcal{M}\left(\mathbb{T}^{*}\right)}\left(h_{2}\left(\mathrm{v}_{1}^{\varepsilon}\right)-h_{2}\left(\mathrm{u}_{1}^{\varepsilon}\right)\right) \cdots\left(h_{2}\left(\mathrm{v}_{m}^{\varepsilon}\right)-h_{2}\left(\mathrm{u}_{m}^{\varepsilon}\right)\right) \mathrm{d} \mu^{\mathbb{T}}\left(\mathrm{L}^{*}\right) \\
= & \left(\mathcal{G}\left(u_{1}, v_{1}, \ldots, u_{k}, v_{k}\right)+\mathrm{O}(\varepsilon)\right) \mathbb{E}_{\mu^{\mathbb{T}}}\left[\left(h_{2}\left(\mathrm{v}_{1}^{\varepsilon}\right)-h_{2}\left(\mathrm{u}_{1}^{\varepsilon}\right)\right) \cdots h_{2}\left(\mathrm{v}_{m}^{\varepsilon}\right)-h_{2}\left(\mathrm{u}_{m}^{\varepsilon}\right)\right] \\
= & \left(\mathcal{G}\left(u_{1}, v_{1}, \ldots, u_{k}, v_{k}\right)+\mathrm{O}(\varepsilon)\right)\left(\mathcal{G}\left(\mathrm{u}_{1}, \mathrm{v}_{1}, \ldots, \mathrm{u}_{m}, \mathrm{v}_{m}\right)+\mathrm{O}(\varepsilon)\right),
\end{aligned}
$$

where the last line is obtained by using Proposition 11 for the graph $\mathbb{T}$.

Lemma 23. When $\varepsilon$ is small, and for every lozenge-with-diagonals tiling $L \in \mathcal{L}$,

$$
\mathbb{E}_{\mu^{L}}\left[\left(h_{1}\left(v_{1}^{\varepsilon}\right)-h_{1}\left(u_{1}^{\varepsilon}\right)\right) \cdots\left(h_{1}\left(v_{k}^{\varepsilon}\right)-h_{1}\left(u_{k}^{\varepsilon}\right)\right)\right]=\mathcal{G}\left(u_{1}, v_{1}, \ldots, u_{k}, v_{k}\right)+\mathrm{O}(\varepsilon),
$$

where $\mathrm{O}(\varepsilon)$ is independent of $L$.

Proof. Let us look at the proof of Proposition 11 in the case of a lozenge-with-diagonals tiling $L \in \mathcal{L}$, and denote by $K_{L}$ the Dirac operator indexed by vertices of $L^{*}$. Then Lemma 23 is proved if we show that $\mathrm{O}(\varepsilon)$ in Lemma 14 
is independent of $L \in \mathcal{L}$. Looking at the proof of Lemma 14, we see that $\mathrm{O}(\varepsilon)$ comes from the error term in the asymptotic formula for the inverse Dirac operator of Theorem 10, [13]:

$$
K_{L}^{-1}\left(b_{i}, w_{j}\right)=\varepsilon\left(\frac{1}{2 \pi}\left(F_{0}\left(b_{i}, w_{j}\right)+f_{w_{j} b_{i}}(0) F_{1}\left(b_{i}, w_{j}\right)\right)+\mathrm{O}\left(\frac{\varepsilon}{|b-w|^{2}}\right)\right) .
$$

In [13], the error term is computed explicitly. Looking at the explicit formula, and using the regularity of the graphs $L$, we show that $\mathrm{O}\left(\varepsilon /|b-w|^{2}\right)$ is in fact equivalent to $C_{1} \varepsilon /|b-w|^{2}$, as $\varepsilon \rightarrow 0$, where $C_{1}$ is independent of $L$. Moreover, by assumption the paths $\gamma_{1}, \ldots, \gamma_{k}$ are disjoint, so that we define:

$$
C_{2}=\inf _{i \neq j} \inf _{\left\{b \in \gamma_{i}, w \in \gamma_{j}\right\}}|b-w|>0,
$$

which is independent of $L$. Hence $\mathrm{O}\left(\varepsilon /|b-w|^{2}\right)$ is equivalent to $C_{1} \varepsilon / C_{2}^{2}$, when $\varepsilon$ is small.

\section{Acknowledgements}

We would like to thank Richard Kenyon for proposing the questions solved in this paper, and for the many enlightening discussions. We are grateful to Erwin Bolthausen, Cédric Boutillier and Wendelin Werner for their advice and suggestions.

\section{References}

[1] S. Bochner, Harmonic Analysis and the Theory of Probabilities, Univ. of California Press, 1960.

[2] O. Bodini, M. Latapy, Generalized tilings with height functions, Morfismos 7 (1) (2003) 47-68.

[3] B. de Tilière, Quadri-tilings of the plane, math.PR/0403324, Probab. Theory Related Fields, in press.

[4] B. de Tilière, Dimères sur les graphes isoradiaux \& modèle d'interfaces aléatoires en dimension 2+2, PhD Thesis, Université Paris XI, Orsay, 2004.

[5] N. Elkies, G. Kuperberg, M. Larsen, J. Propp, Alternating-sign matrices and domino tilings, J. Algebraic Combin. 1 (2) (1992) $111-132$.

[6] J. Glimm, A. Jaffe, Quantum Physics. A Functional Integral Point of View, Springer-Verlag, New York, 1981.

[7] I.M. Guelfand, N.Y. Vilenkin, Les distributions, Tome 4 : Applications de l'analyse harmonique, Dunod, Paris, 1967.

[8] R. Kenyon, Local statistics of lattice dimers, Ann. Inst. H. Poincaré Probab. Statist. 33 (5) (1997) 591-618.

[9] R. Kenyon, An Introduction to the Dimer Model, ICTP Lect. Notes, vol. XVII, 2004.

[10] R. Kenyon, The planar dimer model with boundary: a survey, in: CRM Monogr. Ser., vol. 13, Amer. Math. Soc., Providence, RI, 2000, pp. 307-328.

[11] R. Kenyon, Conformal invariance of domino tilings, Ann. Probab. 28 (2) (2000) 759-795.

[12] R. Kenyon, Dominos and the Gaussian free field, Ann. Probab. 29 (3) (2001) 1128-1137.

[13] R. Kenyon, The Laplacian and Dirac operators on critical planar graphs, Invent. Math. 150 (2) (2002) $409-439$.

[14] R. Kenyon, Height fluctuations in the honeycomb dimer model, math-ph/0405052.

[15] R. Kenyon, A. Okounkov, S. Sheffield, Dimers and amoebas, Ann. of Math. (2) 163 (3) (2006) 1019-1056.

[16] C. Mercat, Discrete period matrices and related topics, math-ph/0111043.

[17] S. Sheffield, Random Surfaces, Asterisque, vol. 304, 2005.

[18] S. Sheffield, Gaussian Free Field for mathematicians, math.PR/0312099.

[19] W.P. Thurston, Conway's tiling groups, Amer. Math. Monthly 97 (8) (1990) 757-773. 